\title{
ON LINEAR VOLTERRA EQUATIONS OF PARABOLIC TYPE IN BANACH SPACES
}

\author{
JAN PRÜSS
}

\begin{abstract}
Linear integrodifferential equations of Volterra type in a Banach space are studied in case the main part of the equation generates an analytic $C_{0}$-semigroup. Under very general assumptions it is shown that a resolvent operator exists and that many of the solution properties of parabolic evolution equations are inherited. The results are then applied to integro-partial differential equations of parabolic type.
\end{abstract}

1. Introduction. Let $X$ be a Banach space and consider the linear initial value problem

$$
\begin{aligned}
& u^{\prime}(t)=A u(t)+\int_{0}^{t} d B(\tau) u(t-\tau)+f(t), \quad t \geq 0, \\
& u(0)=u_{0}
\end{aligned}
$$

Here $A$ denotes a closed linear operator with domain $D(A)$ dense in $X,\{B(t)\}_{t \geq 0}$ a family of closed linear operators in $X$ with domains $D(B(t)) \supset D(A)$, which is of bounded variation in a suitable sense, $f \in L_{\text {loc }}^{1}\left(\mathbf{R}_{+}, X\right)$ and $u_{0} \in X$; see $\S 2$ for more details. (1) is an abstract version of the heat equation in materials with memory (see e.g. Coleman and Gurtin [2])

$$
\begin{aligned}
& u_{t}(t, x)=\Delta u(t, x)+\int_{0}^{t} d b(\tau) \Delta u(t-\tau, x)+f(t, x) \quad \text { on } \mathbf{R}_{+} \times \Omega, \\
& \frac{\partial u}{\partial \nu}(t, x)=0 \quad \text { on } \partial \Omega, \\
& u(0, x)=u_{0}(x) \quad \text { on } \Omega,
\end{aligned}
$$

as well as for many other problems, e.g. from viscoelasticity, rheology or population dynamics; see $\S 5$.

The solution properties of (1) have been studied by numerous authors under various hypotheses on $X, A$ and $B(t)$. The main problem concerning (1) is existence of a resolvent operator, i.e. a family of strongly continuous bounded linear operators $\{S(t)\}_{t \geq 0}$ in $X$ which satisfy $S(0)=I$ (identity) and the resolvent equations

(3) $S^{\prime}(t)=A S(t)+\int_{0}^{t} d B(\tau) S(t-\tau) \quad$ and $\quad S^{\prime}(t)=S(t) A+\int_{0}^{t} S(t-\tau) d B(\tau)$

Received by the editors April 29, 1986.

1980 Mathematics Subject Classification (1985 Revision). Primary 45N05, 45K05; Secondary $34 \mathrm{G} 10,47 \mathrm{D} 05$.

Key words and phrases. Analytic semigroup, Laplace transform, maximal regularity, resolvent operator, resolvent equations, Volterra operator. 
in a suitable sense. It is well known that in case (1) admits a resolvent $S(t)$, any solution of (1) is represented by the variation of parameters formula

$$
u(t)=S(t) u_{0}+\int_{0}^{t} S(t-\tau) f(\tau) d \tau
$$

in particular, the solutions of (1) are uniquely determined by and depend continuously on the data $u_{0}$ and $f$, i.e. (1) is well posed, and also $S(t)$ itself is unique. Conversely, if the homogeneous equation (1) is well posed (in the sense of Phillips [13]) the resolvent operator for (1) exists.

As formula (4) shows, all solution properties of (1) as regularity and growth are reflected in the resolvent operator $S(t)$ of $(1)$, but knowledge of $S(t)$ is also important for several other problems which are related to (1). For instance, if $S(\cdot)$ exists and belongs to $L^{1}\left(\mathbf{R}_{+}, \mathbf{B}(X)\right)$ then

$$
u(t)=\int_{0}^{\infty} S(t-\tau) g(\tau) d \tau, \quad t \in \mathbf{R}
$$

is the unique bounded solution of the infinite delay equation

$$
u^{\prime}(t)=A u(t)+\int_{0}^{\infty} d B(\tau) u(t-\tau)+g(t), \quad t \in \mathbf{R},
$$

where $g \in L^{\infty}(\mathbf{R}, X)$, but also in case $S \notin L^{1}\left(\mathbf{R}_{+}, \mathbf{B}(X)\right)$, important conclusions on the solvability behavior of $\left(1^{\prime}\right)$ on the real line can be drawn from mere existence of $S(t)$; compare [14 and 15]. Also, as in the theory of semilinear evolution equations, formula (4) can be used to rewrite the nonlinearly perturbed problem

$$
\begin{aligned}
& u^{\prime}(t)=A u(t)+\int_{0}^{t} d B(\tau) u(t-\tau)+f(t)+(F u)(t), \quad t \geq 0 \\
& u(0)=u_{0}
\end{aligned}
$$

as an integral equation

$$
u(t)=S(t) u_{0}+\int_{0}^{t} S(t-\tau) f(\tau) d \tau+\int_{0}^{t} S(t-\tau)(F u)(\tau) d \tau
$$

which is in general much easier to handle than the original equation since the unbounded parts have been removed.

There are essentially two different known results on existence of the resolvent. In the so-called hyperbolic case it is assumed that $A$ generates a $C_{0}$-semigroup in $X$ and that $B(\cdot) x \in W_{\text {loc }}^{1,1}\left(\mathbf{R}_{+}, X\right), B^{\prime}(\cdot) x \in \mathrm{BV}_{\text {loc }}\left(\mathbf{R}_{+}, X\right)$ (the space of functions of bounded variation) hold for each $x \in D(A)$; see Grimmer and Prüss [11] for a proof. On the other hand, in the parabolic case $A$ is supposed to generate an analytic $C_{0}$-semigroup and $B(\cdot) x \in C_{\text {loc }}^{1+\alpha}\left(\mathbf{R}_{+}, X\right)$ for each $x \in D(A)$; see Friedman and Shinbrot $[\mathbf{8}]$. While the first result is best possible as counterexamples [11] show, the second one is not and several attempts to improve it have been made, see e.g. Grimmer and Kappel [9], Grimmer and Pritchard [10], Da Prato and Ianelli [5], Da Prato, Ianelli and Sinestrari [6]. However, in each of these papers some regularity of $B(t)$ had to be assumed, either explicitly or implicitly through growth conditions on the Laplace transform of $B(t)$, although the in some sense worst example

$$
u^{\prime}(t)=A u(t)+A u(t-1)+f(t)
$$


indicates that no such assumption is needed. In fact, for this equation the resolvent $S(t)$ is given by

$$
S(t)=\sum_{n=0}^{\infty}(n !)^{-1}(t-n)_{+}^{n} \cdot A^{n} e^{A(t-n)_{+}}, \quad(a)_{+}=\max (a, 0),
$$

as a simple computation shows; evidently $S(t)$ is strongly continuous and bounded since $t A e^{A t}$ is bounded.

Our main purpose in this paper is to prove that in the parabolic case there is always a resolvent operator for (1), no further regularity of $B(\cdot)$ is needed. Even more, we show that many of the solution properties of $u^{\prime}=A u+f$ are inherited on (1); for instance, if $f \in C_{\mathrm{loc}}^{\alpha}\left(\mathbf{R}_{+}, X\right)$ and $u_{0}=f(0)=0$ then the solution $u$ of (1) satisfies $u^{\prime}, A u \in C_{\mathrm{loc}}^{\alpha}\left(\mathbf{R}_{+}, X\right)$; and if $f \in \mathrm{BV}_{\text {loc }}\left(\mathbf{R}_{+}, X\right)$ then $u, A u \in C\left(\mathbf{R}_{+}, X\right)$ and $u^{\prime} \in L_{\text {loc }}^{\infty}\left(\mathbf{R}_{+}, X\right)$ is continuous except for at most countable many jumps. However, unless $B(\cdot)$ is smooth, $S(t)$ does not share all of the smoothness properties $e^{A t}$ enjoys, as can be seen from the above example, too. This $S(t)$ is not uniformly continuous at $t \in \mathbf{N}_{0}, A S(t)$ becomes unbounded at these points, and $A S(\cdot)$ does not even belong to $L_{\text {loc }}^{1}((0, \infty), \mathbf{B}(X))$. In fact, the singularity $t=0$ of $e^{A t}$ is reproduced in $S(t)$ by the jump points of $\mathbf{B}(\cdot)$, and we show that this is also true for the general equation (1); as a rule, $S(\cdot)$ will be as smooth as $B(\cdot)$.

2. Definitions and main results. Let $X$ be a Banach space with norm $|\cdot|$ and let $A$ denote a (fixed) closed linear and densely defined operator in $X$. Then $Y=D(A)$ equipped with the graph norm $|x|_{Y}=|x|+|A x|$ of $A$ is a Banach space, too, continuously and densely embedded into $X$. Let $\mathbf{B}(Y, X)$ denote the space of all bounded linear operators $T: Y \rightarrow X$ with norm $|T|_{Y, X}=\sup \left\{|T x|:|x|_{Y} \leq 1\right\}$, and $\mathbf{B}(X)=\mathbf{B}(X, X)$. For any interval $J \subset \mathbf{R}$, the spaces $C(J, X)$ of continuous $X$-valued functions on $J, C^{\alpha}(J, X)$ of $\alpha$-Hölder-continuous functions $(\alpha \in(0,1))$, $L^{p}(J, X)$ of $p$-summable functions $(p \in[1, \infty])$ are defined as usual and the norms are denoted by $|\cdot|_{X, 0},|\cdot|_{X, \alpha},|\cdot|_{X, p}$, respectively.

Throughout all of this paper the family $\{B(t)\}_{t \geq 0}$ of closable linear operators in $X$ is supposed to satisfy the following condition:

(B) For all $t \geq 0$ the relation $D(B(t)) \supset D(A)$ holds; $B(0)=B(0+)=0$, $B \in \mathrm{BV}(J, \mathbf{B}(Y, X))$ for each interval $J=[0, a]$ and w.i.o.g. $B$ is left-continuous on $\mathbf{R}_{+}=[0, \infty)$ in $\mathbf{B}(Y, X)$.

Here BV means that the total variation $b(a)=\left.\operatorname{Var} B\right|_{0} ^{a}$ of $B$ on $J$ in $\mathbf{B}(Y, X)$ defined by

$$
b(a)=\sup \left\{\sum_{1}^{n}\left|B\left(t_{i}\right)-B\left(t_{i-1}\right)\right|_{Y, X}: 0=t_{0}<t_{1}<\cdots<t_{n}=a\right\}
$$

is finite. Note that $b$ is increasing, left-continuous with $b(0)=b(0+)=0$ and

$$
|B(t) x-B(s) x| \leq(b(t)-b(s))|x|_{Y}, \quad 0 \leq s \leq t, x \in Y
$$

holds. For $u \in C(J, Y)$ the convolution integral

$$
(d B * u)(t)=\int_{0}^{t} d B(\tau) u(t-\tau), \quad t \in J
$$


exists as a Riemann-Stieltjes integral, it is left-continuous and bounded by

$$
|d B * u|_{X, 0} \leq \sup _{t \in J} \int_{0}^{t} d b(\tau)|u(t-\tau)|_{Y} \leq b(a)|u|_{Y, 0}
$$

$d B * u$ is continuous in case $u(0)=0$ or $B \in C(J, \mathbf{B}(Y, X))$ holds.

Definition 1. Let $f: J \rightarrow X$ be given. A function $u \in C(J, X)$ is called a solution of (1) if $u \in W^{1,1}(J, X) \cap C(J, Y), u(0)=u_{0}$, and (1) holds for a.a. $t \in J$.

Note that even in case $f$ is smooth, a solution of (1) will in general not admit a continuous derivative since $d B * u$ is not continuous unless $u_{0}=0$ or $B$ is $\mathbf{B}(Y, X)$ continuous.

DEFINITION 2. A family of bounded linear operators $\{S(t)\}_{t \geq 0} \subset \mathbf{B}(X)$ is called a resolvent operator or resolvent for (1) if

(S1) For each $x \in X, S(t) x$ is continuous on $\mathbf{R}_{+}$and $S(0) x=x$.

(S2) $S(t) D(A) \subset D(A)$ holds for all $t \geq 0$; for $x \in D(A), A S(t) x$ is continuous and $S(t) x$ locally Lipschitz and a.e. differentiable on $\mathbf{R}_{+}$.

(S3) For each $x \in D(A)$ and a.a. $t \geq 0$, the resolvent equations

$$
\begin{aligned}
& S^{\prime}(t) x=A S(t) x+\int_{0}^{t} d B(\tau) S(t-\tau) x, \\
& S^{\prime}(t) x=S(t) A x+\int_{0}^{t} S(t-\tau) d B(\tau) x
\end{aligned}
$$

are satisfied.

It should be mentioned that the convolution integral in the second resolvent equation does not make sense as a Riemann-Stieltjes integral since $S(t)$ is only assumed to be strongly continuous. However, there are no problems with its integrated form

$$
S(t) x=x+U(t) A x+\int_{0}^{t} U(t-\tau) d B(\tau) x,
$$

where $U(t)=\int_{0}^{t} S(\tau) d \tau$, and so the second resolvent equation will be understood in this version in the sequel.

The first easy consequences of these definitions are the variation of parameters formula (4) and the uniqueness of resolvents.

Proposition 1. Suppose $S(t)$ is a resolvent for $(1) ;$ let $u_{0} \in X$ and $f \in$ $L^{1}(J, X)$, where $J=[0, a]$. Then, if $u(t)$ is a solution of $(1)$ on $J$, we have

$$
u(t)=S(t) u_{0}+\int_{0}^{t} S(t-\tau) f(\tau) d \tau \quad \text { for all } t \in J .
$$

In particular, there is at most one resolvent operator for (1).

Conversely, formula (4) defines a function $u \in C(J, X)$, for any $u_{0} \in X$ and $f \in L^{1}(J, X)$, which is called the mild solution of (1). The next two propositions answer the question when $u$ will be a solution of (1) in the sense of Definition 1 . It is obvious that $u_{0}$ must belong to $D(A)$ then.

Proposition 2. Suppose $S(t)$ is a resolvent for $(1)$ and let $x \in D(A)$. Then $u(t)=S(t) x$ has the following properties.

(i) $u \in C(J, Y) \cap W^{1, \infty}(J, X)$ for each interval $J=[0, a]$, and $u^{\prime}$ is continuous except for jumps at points in $N(x)=\{t>0: B(t+) x \neq B(t) x\}$; 
(ii) $u$ is left-differentiable and $u_{-}^{\prime}(t)=A u(t)+\int_{0}^{t} d B(\tau) u(t-\tau)$ holds for each $t>0$

(iii) $u$ is right-differentiable and $u_{+}^{\prime}(t)-u_{-}^{\prime}(t)=B(t+)-B(t) x$ holds for each $t>0$.

In particular, $u \in C^{\mathbf{1}}(J, X)$ iff $B(\cdot) x$ is continuous on $J$.

Proposition 3. Suppose $S(t)$ is a resolvent for (1) and let $f \in C(J, X)$. Then for $u=S * f \in C(J, X)$ the following are equivalent

(i) $u$ is a solution of (1);

(ii) $u \in C^{1}(J, X)$;

(iii) $u \in C(J, Y)$.

Moreover, if $f \in W^{1,1}(J, X) \cup C(J, Y)$ then $u$ is a solution of (1).

Proofs for these results can be found in $[\mathbf{1 1}]$ for the case of a differentiable $B(t)$ and in $[\mathbf{1 4}]$ for the general case.

Propositions 1 to 3 show that existence of resolvents is the main question concerning (1). It can be answered in the affirmative if $A$ generates an analytic semigroup in $X$, without any further assumption on $B$, besides (B) of course.

THEOREM 1. Let $A$ be the generator of an analytic $C_{0}$-semigroup $T(t)$ in $X$. Then, there exists a resolvent operator $S(t)$ for $(1)$. $S(t)$ has the following additional properties.

(i) $S \in L^{\infty}(J, \mathbf{B}(X)) \cap L^{\infty}(J, \mathbf{B}(Y)) \cap W^{1, \infty}(J, \mathbf{B}(Y, X))$ for each $J=[0 ; a]$;

(ii) $S(t)$ is left-continuous in $\mathbf{B}(X)$ and in $\mathbf{B}(Y)$ on $(0, \infty)$;

(iii) $S(t)$ is right-continous in $\mathbf{B}(X)$ and $\mathbf{B}(Y)$ on $(0, \infty) \backslash N_{r}$, where $N_{r}$ denotes the set of jump points of the resolvent kernel $r$ of $b$. In particular, $S(t)$ is continuous in $\mathbf{B}(X)$ and $\mathbf{B}(Y)$ on $(0, \infty)$ in case $B$ is $\mathbf{B}(Y, X)$-continuous on $\mathbf{R}_{+}$.

Note that the resolvent kernel of $b$ is the solution of $r=b+d b * r$, hence $N_{r}=\{t>0: r(t) \neq r(t+)\}$ is the semimodule generated by the jump points of $b$, i.e. $N=\{t>0: b(t) \neq b(t+)\}$. The example in the introduction shows that Theorem 1 is optimal.

The proof of Theorem 1 is quite involved and therefore postponed to $\S 4$. It is based on the Neumann-series for $S(t)$,

$$
S=T+T * d B * T+T * d B * T * d B * T+\cdots,
$$

which at this point makes sense only formally, since $A T$ is in general not of bounded variation, neither in $\mathbf{B}(X)$ nor in the strong sense. The crucial step in the proof consists in deriving a term by term estimate of this series to obtain the uniform boundedness of certain approximations of $S$, and then also of $S$.

Our next results, somewhat surprising at first sight, show that the solutions of (1) with $u_{0}=0$ essentially exhibit the same regularity as those of the differential equation $u^{\prime}=A u+f$. But first we have to recall the definition of the intermediate spaces $Y_{\alpha}=D_{A}(\alpha)$ of $A$ introduced by DaPrato and Grisvard [4]. For $\alpha \in(0,1)$ we have

$$
Y_{\alpha}=D_{A}(\alpha)=\left\{x \in X: t^{-\alpha}(T(t) x-x) \rightarrow 0 \text { as } t \rightarrow 0\right\}
$$

and for $x \in Y_{\alpha}$

$$
|x|_{\alpha}=|x|+\sup \left\{\bar{t}^{\alpha}|T(t) x-x|: 0<t \leq 1\right\}
$$


then $Y_{\alpha}$ is a Banach space w.r. to the norm $|\cdot|_{\alpha}$ and

$$
Y \hookrightarrow Y_{\alpha} \hookrightarrow Y_{\beta} \hookrightarrow X
$$

holds for $1>\alpha>\beta>0$, where all inclusions are continuous and dense; moreover, we let

$$
Y_{1+\alpha}=D_{A}(\alpha+1)=\left\{x \in Y_{\alpha}: A x \in Y_{\alpha}\right\}, \quad|x|_{1+\alpha}=|x|_{\alpha}+|A x|_{\alpha} .
$$

Since the spaces $Y_{\alpha}$ are interpolation spaces, Theorem 1 immediately implies

COROLlaRY 1. In the situation of Theorem 1 , for $\alpha \in(0,1)$, the resolvent $S(t)$ is also strongly continuous in $Y_{\alpha}$, left-continuous in $\mathbf{B}\left(Y_{\alpha}\right)$ on $(0, \infty)$, and right-continuous in $\mathbf{B}\left(Y_{\alpha}\right)$ on $(0, \infty) \backslash N_{r}$.

If $B$ preserves spatial regularity in the sense that $B$ also belongs to $\mathrm{BV}_{\text {loc }}\left(\mathbf{R}_{+}, \mathbf{B}\left(Y_{1+\alpha}, Y_{\alpha}\right)\right)$, then $S(t)$ is also a resolvent in $Y_{\alpha}$; apply Theorem 1 with $X=Y_{\alpha}$ to see this. However, in general neither $A S(t)$ nor $S^{\prime}(t)$ map $Y_{1+\alpha}$ to $Y_{\alpha}$ and so Corollary 1 cannot be improved; see $\S 5$ for an example.

THEOREM 2. Let $A$ be the generator of an analytic $C_{0}$-semigroup $T(t)$ in $X$, and, for some $\alpha \in(0,1)$, suppose

(i) $f \in C^{\alpha}(J, X)$ and $f(0) \in Y_{\alpha}$; or

(ii) $f \in C\left(J, Y_{\alpha}\right)$.

Then $u=S * f$ is the solution of (1) with $u_{0}=0$ and additionally satisfies in case (i)

$$
u \in C^{\alpha}(J, Y) \cap C^{1+\varepsilon}\left(J, Y_{\alpha-\varepsilon}\right) \quad \text { for each } \varepsilon \in(0, \alpha]
$$

and in case (ii)

$$
u \in C^{\alpha}(J, Y) \cap C^{1}\left(J, Y_{\alpha-\varepsilon}\right) \quad \text { for each } \varepsilon \in(0, \alpha] .
$$

Note that this result is optimal in case (i); in case (ii) the assertion can only be improved in case $B(t)$ preserves spatial regularity. By means of a simple interpolation argument, it is possible to prove that in any case $u \in C^{\beta}\left(J, Y_{\gamma}\right)$ for $\beta+\gamma \leq 1+\alpha, \beta, \gamma \leq 1$ and $(\beta, \gamma) \neq(1, \alpha)$ holds. Let us also note that the fractional power spaces $Y^{\alpha}=D\left((\omega-A)^{\alpha}\right)$ are continuously embedded into $Y_{\alpha}$, hence the case (ii) holds also for $f \in C\left(J, Y^{\alpha}\right)$.

A weaker type of regularity which also includes nonvanishing initial values $u_{0}$ of $u$ involves the following function spaces. For $\alpha \in(0,1)$ we define

$$
B^{\alpha}(J, X)=\left\{f \in L^{1}(J, X):[f]_{\alpha}<\infty\right\}
$$

where

$$
[f]_{\alpha}=\sup _{h>0} h^{-\alpha} \int_{0}^{a-h}|f(t+h)-f(t)| d t+\sup _{h>0} h^{-\alpha} \int_{0}^{\alpha} \log \left(1+\frac{h}{t}\right)|f(t)| d t ;
$$

obviously $B^{\alpha}(J, X) \supset C^{\alpha}(J, X)$ holds. For this class we have

THEOREM 3. Let $A$ be the generator of an analytic $C_{0}$-semigroup $T(t)$ in $X$, and suppose $f \in B^{\alpha}(J, X)$ and $u_{0} \in Y_{\alpha}$ for some $\alpha \in(0,1)$. Then $u=S u_{0}+S * f$ satisfies $u \in W^{1,1}(J, X) \cap L^{1}(J, Y)$ and $u^{\prime}, A u \in B^{\alpha}(J, X)$, and equation (1) holds for a.a. $t \in J$.

Here the convolution $d B * u$ has to be understood in $L^{1}(J, X)$, as will be discussed in the next section where the proofs of Theorems 2 and 3 are given. Let us note 
that the result of Theorem 3 is very flexible since the assumptions are very weak. It will prove its usefulness also for the proof of Theorem 1 in $\S 4$, in particular since nonvanishing initial values are admitted.

Finally, we state the following regularity result which is well known for differential equations $u^{\prime}=A u+f$; see Webb [18].

THEOREM 4. Let $A$ be the generator of an analytic $C_{0}$-semigroup $T(t)$ in $X$, and let $f \in B V(J, X)$. Then $u=S * f \in C(J, Y) \cap W^{1, \infty}(J, X)$ is the solution of (1) with $u_{0}=0$; furthermore $u^{\prime}(t)$ is left-continuous on $J$, right-continuous at all points of continuity of $f$ and $u^{\prime}(t+)-u^{\prime}(t)=f(t+)-f(t)$ holds for all $t \in J$.

The proof of this result is easy, once it is shown that

$$
u^{\prime}(t)-f(t)=\int_{0}^{t}(S(t-\tau)-I) d f(\tau)+(S(t)-I) f(0)
$$

exists and is continuous; this in turn will follow by the same method which yields Theorem 1.

3. Proofs of Theorem 2 and 3. The proofs of Theorem 2 and 3 are based on properties of the analytic semigroup $T(t)$ generated by $A$, in particular of the solutions of the initial value problem

$$
\begin{aligned}
& v^{\prime}(t)=A v(t)+g(t), \quad t \geq 0, \\
& v(0)=0
\end{aligned}
$$

The fundamental property of such semigroups is

$$
\begin{aligned}
& T(t) X \subset D(A) \quad \text { for all } t>0, \quad \text { and } \\
& |T(t)| \leq M, \quad|A T(t)| \leq M / t \quad \text { for } t \in(0, a],
\end{aligned}
$$

where the constant $M$ may depend on $a$. It is well known that (6) is characterizing analytic $C_{0}$ semigroups.

For $\alpha \in(0,1)$ it is easy to see that $\left.T(t)\right|_{Y_{\alpha}}$ is also an analytic $C_{0}$-semigroup in $Y_{\alpha}$ and estimates (6) in $Y_{\alpha}$ hold with the same constant $M$. The strong continuity of $T(t)$ at $t=0$ in $Y_{\alpha}$ follows since $\left\{t^{-\alpha}(T(t) x-x): t \in(0,1]\right\}$ is relatively compact in $X$ for $x \in Y_{\alpha}$. It is also easy to see that $Y_{\alpha}$ is characterized by

$$
Y_{\alpha}=\left\{x \in X: t^{1-\alpha} A T(t) x \rightarrow 0 \text { for } t \rightarrow 0\right\}
$$

and

$$
\|x\|_{\alpha}=|x|+\sup _{t \leq a} t^{1-\alpha}|A T(t) x|
$$

is a norm on $Y_{\alpha}$ equivalent to $|\cdot|_{\alpha}$; more precisely,

$$
(2 M)^{-1}\|x\|_{\alpha} \leq|x|_{\alpha} \leq \alpha^{-1}\|x\|_{\alpha} .
$$

To state the solution properties of (5) needed below, we have to introduce the norms $|\cdot|_{\alpha, \beta}$ on the spaces $C^{\beta}\left(J, Y_{\alpha}\right)$ of Hölder-continuous functions $f: J \rightarrow Y$, where $(\alpha, \beta) \in[0,1] \times[0,1)$.

$$
|f|_{\alpha, 0}=\sup _{J}|f(t)|_{\alpha} \quad \text { for } \alpha \in[0,1]
$$


and

$$
|f|_{\alpha, \beta}=|f|_{\alpha, 0}+\sup _{t \neq \bar{t}}|f(t)-f(\bar{t})|_{\alpha} \cdot|t-\bar{t}|^{-\beta} \quad \text { for } \alpha \in[0,1], \beta \in(0,1)
$$

here $\alpha=0,1$ refer to $Y_{0}=X$ and to $Y_{1}=Y$, respectively.

The first lemma deals with the properties of the solution of (5) in case $g$ is Hölder-continuous; see Sinestrari [16].

LEMMA 1. Suppose $g \in C^{\alpha}(J, X)$ and $A v_{0}+g(0) \in Y_{\alpha}$ for some $\alpha \in(0,1)$, and let $v(t)=T(t) v_{0}+(T * g)(t)$ for $t \in J$. Then $v(t)$ is the solution of $(5)$ in $J$ and satisfies

$$
v \in C^{\alpha}(J, Y) \cap C^{1+\varepsilon}\left(J, Y_{\alpha-\varepsilon}\right) \cap C^{1+\alpha}(J, X) \quad \text { for each } \varepsilon \in(0, \alpha),
$$

and

$$
|v|_{1, \alpha}+\left|v^{\prime}\right|_{\alpha, 0}+\left|v^{\prime}\right|_{0, \alpha} \leq C \cdot\left(\left|A v_{0}+g(0)\right|_{\alpha}+|g|_{0, \alpha}\right)
$$

where $C=C(\alpha, a, M)$ denotes a constant only depending on $\alpha, a, M$.

Proof. It is well known that $v \in C^{1}(J, X) \cap C(J, Y)$ is the unique solution of (5), we therefore need only to verify inequality (7). Let

$$
v_{1}(t)=T(t) v_{0}+(T * g(0))(t)=T(t) v_{0}+\int_{0}^{t} T(\tau) d \tau g(0) ;
$$

then $A v_{1}(t)=T(t)\left(A v_{0}+g(0)\right)=v_{1}^{\prime}(t)-g(0)$. The asserted inequality for $v_{1}$ is now a trivial consequence of the definition of $Y_{\alpha}$ and the strong continuity of $\left.T(t)\right|_{Y_{\alpha}}$. Therefore, we may assume $v_{0}=g(0)=0$ in the sequel.

To verify the inequality for $\left|v^{\prime}\right|_{0, \alpha}$ we decompose according to

$$
\begin{aligned}
v^{\prime}(t)-v^{\prime}(\bar{t})= & (T(t)-T(\bar{t}))(g(\bar{t})-g(0))+T(h)(g(t)-g(\bar{t})) \\
& +\int_{\bar{t}}^{t} A T(t-s)(g(s)-g(t)) d s \\
& +\int_{0}^{t} A(T(h+s)-T(s))(g(\bar{t}-s)-g(\bar{t})) d s \\
= & I_{1}+I_{2}+I_{3}+I_{4},
\end{aligned}
$$

where $t>\bar{t}$ and $h=t-\bar{t}$, and estimate separately

$$
\begin{aligned}
\left|I_{1}\right| & \leq|T(t)-T(\bar{t})||g(\bar{t})-g(0)| \leq M|g|_{0, \alpha} \bar{t}^{\alpha} \int_{\bar{t}}^{t} \tau^{-1} d \tau \\
& \leq M|g|_{0, \alpha} \alpha^{-1} h^{\alpha} ; \\
\left|I_{2}\right| & \leq|T(h)||g(t)-g(\bar{t})| \leq M|g|_{0, \alpha} h^{\alpha} ; \\
\left|I_{3}\right| & \leq \int_{\bar{t}}^{t}|A T(t-s)||g(s)-g(t)| d s \leq M|g|_{0, \alpha} \int_{\bar{t}}^{t}(t-s)^{\alpha-1} d s \\
& =M|g|_{0, \alpha} \alpha^{-1} h^{\alpha} ; \\
\left|I_{4}\right| & \leq \int_{0}^{\bar{t}} \int_{s}^{s+h}\left|A^{2} T(r)\right| d r|g(\bar{t}-s)-g(\bar{t})| d s \\
& \leq 4 M^{2}|g|_{0, \alpha} \int_{0}^{\bar{t}} h s^{\alpha-1}(s+h)^{-1} d s \leq 4 M^{2}|g|_{0, \alpha} h^{\alpha} \int_{0}^{\infty} \tau^{\alpha-1}(1+\tau)^{-1} d \tau \\
& =4 \pi M^{2}|g|_{0, \alpha}(\sin \alpha \pi)^{-1} h^{\alpha} .
\end{aligned}
$$


This shows $v^{\prime} \in C^{\alpha}(J, X)$ and $\left|v^{\prime}\right|_{0, \alpha} \leq C|g|_{0, \alpha}$, hence by (5) we also obtain $v \in C^{\alpha}(J, Y)$ and $|v|_{1, \alpha} \leq C|g|_{0, \alpha}$. Further,

$$
v^{\prime}(t)=\int_{0}^{t} A T(s)(g(t-s)-g(t)) d s+T(t) g(t)
$$

yields

$$
\begin{aligned}
\left\|v^{\prime}(t)\right\|_{\alpha} \leq & \left|v^{\prime}(t)\right| \\
& +\sup _{\tau \leq a} \tau^{1-\alpha}\left\{\int_{0}^{t}\left|A^{2} T(s+\tau)\right||g(t-s)-g(t)| d s+|A T(t+\tau)||g(t)|\right\} \\
\leq & C|g|_{0, \alpha}\left\{1+\sup _{\tau \leq a} \tau^{1-\alpha}\left(\int_{0}^{t} s^{\alpha}(s+\tau)^{-2} d s+t^{\alpha}(t+\tau)^{-1}\right)\right\} \\
\leq & C|g|_{0, \alpha}\left\{2+\int_{0}^{\infty} r^{\alpha}(1+r)^{-2} d r\right\} \\
= & C|g|_{0, \alpha}\left\{2+\pi \alpha(\sin \alpha \pi)^{-1}\right\} .
\end{aligned}
$$

Hence $\left|v^{\prime}\right|_{\alpha, 0} \leq C|g|_{0, \alpha}$ holds for some constant $C$. This estimate already implies $v^{\prime}(t) \in Y_{\alpha-\varepsilon}$ for each $t \in J, \varepsilon \in(0, \alpha)$, and the remaining assertion $v^{\prime} \in C^{\varepsilon}\left(J, Y_{\alpha-\varepsilon}\right)$ now follows by a simple interpolation argument. In fact, with

$$
\varphi(t, \tau, h)=\tau^{1-\alpha+\varepsilon}\left|A T(\tau)\left(v^{\prime}(t+h)-v^{\prime}(t)\right)\right|
$$

we have

$$
\varphi(t, \tau, h) \leq M \tau^{\varepsilon-\alpha} h^{\alpha}\left|v^{\prime}\right|_{0, \alpha}
$$

as well as

$$
\varphi(t, \tau, h) \leq 2 \tau^{\varepsilon}\left|v^{\prime}\right|_{\alpha, 0}
$$

and therefore

$$
\begin{aligned}
\left\|v^{\prime}(t+h)-v^{\prime}(t)\right\|_{\alpha-\varepsilon} & \leq C\left|v^{\prime}\right|_{0, \alpha} \cdot h^{\alpha}+\sup _{\tau \leq a} \varphi(t, \tau, h) \\
& \leq C\left|v^{\prime}\right|_{0, \alpha} \cdot h^{\alpha}+\sup _{\tau \leq a}\left(\varphi(t, \tau, h)^{(1-\varepsilon / \alpha)} \cdot \varphi(t, \tau, h)^{\varepsilon / \alpha}\right) \\
& \leq C\left|v^{\prime}\right|_{0, \alpha} \cdot h^{\alpha}+\left(M\left|v^{\prime}\right|_{0, \alpha}\right)^{\varepsilon / \alpha}\left(2\left|v^{\prime}\right|_{\alpha, 0}\right)^{1-\varepsilon / \alpha} h^{\varepsilon} \\
& \leq C|g|_{0, \alpha} h^{\varepsilon} .
\end{aligned}
$$

It can be shown that $v \in C^{1}\left(J, Y_{\alpha}\right)$ also holds in case $g$ is "little" Hölder-continuous, i.e. $(g(t)-g(t+h)) h^{-\alpha} \rightarrow 0$ as $h \rightarrow 0$. Since we shall not use this concept, we refer to the paper of Sinestrari [16].

LEMma 2. Suppose $g \in C\left(J, Y_{\alpha}\right)$ and $A v_{0} \in Y_{\alpha}$ for some $\alpha \in(0,1)$, and let $v(t)=T(t) v_{0}+(T * g)(t)$ for $t \in J$. Then $v(t)$ is the solution of (5) in $J$ and it satisfies $v \in C\left(J, Y_{1+\alpha}\right) \cap C^{\alpha}(J, Y) \cap C^{1}\left(J, Y_{\alpha}\right)$ and

$$
|A v|_{\alpha, 0}+|v|_{1, \alpha}+\left|v^{\prime}\right|_{\alpha, 0} \leq C\left(\left|A v_{0}\right|_{\alpha}+|g|_{\alpha, 0}\right)
$$

where $C=C(\alpha, a, M)$ denotes a constant only depending on $\alpha, a, M$.

This result is due to Da Prato and Grisvard [4]; we include a proof here for the sake of completeness. 
PROOF. Again we may assume $v_{0}=0$, since $v_{1}(t)=T(t) v_{0}$ satisfies all assertions by definition of $Y_{\alpha}$. Firstly, we have

$$
|A v(t)| \leq \int_{0}^{t}|A T(\tau) g(t-\tau)| d \tau \leq C|g|_{\alpha, 0} \int_{0}^{t} \tau^{\alpha-1} d \tau=C \alpha^{-1} a^{\alpha}|g|_{\alpha, 0}
$$

hence $v(t) \in D(A)$ for all $t \in J$, and $|v|_{1,0} \leq C|g|_{\alpha, 0}$.

Secondly,

$$
\begin{aligned}
\mid A v( & +h)-A v(t) \mid \\
& \leq \int_{t}^{t+h}|A T(t+h-s) g(s)| d s+\int_{0}^{t}\left|\int_{t-s}^{t+h-s} A^{2} T(r) g(s) d r\right| d s \\
& \leq C|g|_{\alpha, 0}\left(\int_{t}^{t+h}(t+h-s)^{\alpha-1} d s+\int_{0}^{t} \int_{t-s}^{t+h-s} r^{\alpha-2} d r d s\right) \\
& \leq C|g|_{\alpha, 0}\left(h^{\alpha} \alpha^{-1}+(1-\alpha)^{-1} \int_{0}^{t}\left[(t-s)^{\alpha-1}-(t+h-s)^{\alpha-1}\right] d s\right) \\
& \leq 2 C|g|_{\alpha, 0} h^{\alpha}(\alpha(1-\alpha))^{-1} ;
\end{aligned}
$$

i.e. $v \in C^{\alpha}(J, Y)$ and $|v|_{1, \alpha} \leq C|g|_{\alpha, 0}$.

Thirdly,

$$
\begin{aligned}
|A v|_{\alpha, 0} & \leq|A v|_{0,0}+\sup _{t, \tau \leq a} \tau^{1-\alpha} \int_{0}^{t}\left|A^{2} T(s+\tau) g(t-s)\right| d s \\
& \leq C|g|_{\alpha, 0}+C|g|_{\alpha, 0} \cdot \sup _{t, \tau \leq a} \tau^{1-\alpha} \int_{0}^{t}(\tau+s)^{\alpha-2} d s \\
& \leq C|g|_{\alpha, 0}\left(1+\int_{0}^{\infty}(1+s)^{\alpha-2} d s\right)=C|g|_{\alpha, 0}\left(1+(1-\alpha)^{-1}\right)
\end{aligned}
$$

and therefore by (5) also $\left|v^{\prime}\right|_{\alpha, 0} \leq C|g|_{\alpha, 0}$.

To show that $A v(t) \in Y_{\alpha}$ for each $t \in J$, we decompose

$$
\begin{aligned}
A v(t)= & (T(\varepsilon)-I) g(t)+T(\varepsilon) \int_{\varepsilon}^{t} A T(s) g(t-s) d s \\
& +\int_{0}^{\varepsilon} A T(s)(g(t-s)-g(s)) d s
\end{aligned}
$$

since the first two terms on the right-hand side of this equation already belong to $Y_{\alpha}$ we obtain

$$
\begin{aligned}
\lim _{\varepsilon \rightarrow 0}\left|\tau^{1-\alpha} A T(\tau) A v(t)\right| & \leq \lim _{\tau \rightarrow 0} \tau^{1-\alpha} \int_{0}^{\varepsilon}\left|A^{2} T(\tau+s)(g(t-s)-g(t))\right| d s \\
& \leq C \sup _{s \leq \varepsilon}|g(t-s)-g(t)|_{\alpha} \cdot \lim _{\tau \rightarrow 0} \tau^{1-\alpha} \int_{0}^{\infty}(\tau+s)^{\alpha-2} d s \\
& \leq C \sup _{s \leq \varepsilon}|g(t-s)-g(t)|_{\alpha} \cdot(1-\alpha)^{-1} \rightarrow 0 \text { as } \varepsilon \rightarrow 0
\end{aligned}
$$

uniformly on $J$, since $g \in C\left(J, Y_{\alpha}\right)$. Hence $A v(t) \in Y_{\alpha}$, and $v^{\prime}(t)=A v(t)+g(t) \in Y_{\alpha}$ for each $t \in J$. 
Finally, to show that $A v$ and $v^{\prime}$ are continuous in $Y_{\alpha}$, write $A v(t+h)-A v(t)=$ $(T(h)-I)\left(v(t)+g(t)+\int_{0}^{h} A T(s)(g(t+h-s)-g(t)) d s\right.$. Since $v(t), g(t) \in Y_{\alpha}$, the first term on the right-hand side of this equation tends to zero as $h \rightarrow 0$. The second one is majorized by

$$
\begin{aligned}
& \left|\tau^{1-\alpha} A T(\tau) \int_{0}^{h} A T(s)(g(t+h-s)-g(t)) d s\right| \\
& \quad \leq C \sup _{s \leq h}|g(t+s)-g(t)|_{\alpha} \cdot \tau^{1-\alpha} \int_{0}^{\infty}(\tau+s)^{\alpha-2} d s \\
& \quad \leq C \sup _{s \leq h}|g(t+s)-g(t)|_{\alpha}(1-\alpha)^{-1} \rightarrow 0 \quad \text { as } h \rightarrow 0 .
\end{aligned}
$$

Thus $A v(t)$ and $v^{\prime}(t)$ are right-continuous in $Y_{\alpha}$; left-continuity follows similarly.

Lemma 2 shows in particular that the intermediate spaces $Y_{\alpha}$ enjoy the property that for any $u_{0} \in Y_{\alpha}$ with $A v_{0} \in Y_{\alpha}$, i.e. $v_{0} \in D\left(\left.A\right|_{Y_{\alpha}}\right)$, and for any $g \in C\left(J, Y_{\alpha}\right)$, the function $v(t)=T(t) v_{0}+(T * g)(t)$ is a solution of (5) in $Y_{\alpha}$; this property is sometimes called the maximal regularity property.

For the class $B^{\alpha}(J, X)$ introduced in $\S 2$ we have the following result which seems to be new. It will be important for the proof of Theorem 3 but also for that of Theorem 1.

LEMMA 3. Suppose $g \in B^{\alpha}(J, X)$ and $v_{0} \in Y_{\alpha}$ for some $\alpha \in(0,1)$, and let $v(t)=T(t) v_{0}+(T * g)(t)$ for $t \in J$. Then $v(t)$ is an a.e. solution of (5) and it satisfies $v \in B^{\alpha}(J, Y) \cap B^{1+\alpha}(J, X)$ and

$$
[A v]_{\alpha}+\left[v^{\prime}\right]_{\alpha} \leq C(\alpha \sin \pi \alpha)^{-1}\left(\left|v_{0}\right|_{\alpha}+[g]_{\alpha}\right),
$$

where $C$ denotes a constant only depending on $M$.

Proof. 1. It suffices to show $A v \in B^{\alpha}(J, X)$ and to derive the estimate for $[A v]_{\alpha}$, since (5) then will imply that for $v^{\prime}$. We may further assume $v_{0}=0$ since $v_{1}(t)=T(t) v_{0}$ satisfies

$$
\begin{aligned}
& \int_{0}^{a} \log \left(1+\frac{h}{t}\right)\left|A v_{1}(t)\right| d t \leq\left|v_{0}\right|_{\alpha} \int_{0}^{a} t^{\alpha-1} \log \left(1+\frac{h}{t}\right) d t \\
& \leq\left|v_{0}\right|_{\alpha} h^{\alpha} \int_{0}^{\infty} s^{\alpha-1} \log \left(1+\frac{1}{s}\right) d s=\pi(\alpha \sin \alpha \pi)^{-1}\left|v_{0}\right|_{\alpha} h^{\alpha}
\end{aligned}
$$

as well as

$$
\begin{aligned}
\int_{0}^{a-h}\left|A v_{1}(t+h)-A v_{1}(t)\right| d t & \leq 4 M\left|v_{0}\right|_{\alpha} \int_{0}^{a-h} \int_{0}^{t+h} s^{\alpha-2} d s d \tau \\
& \leq 4 M\left|v_{0}\right|_{\alpha}(\alpha(1-\alpha))^{-1} h^{\alpha}
\end{aligned}
$$

hence $v_{1}$ belongs to $B^{\alpha}(J, Y) \cap B^{1+\alpha}(J, X)$ and satisfies (9).

2. Let $\rho_{\varepsilon}$ denote real-valued $C^{\infty}$-functions with support supp $\rho_{\varepsilon} \subset(0, \varepsilon), \rho_{\varepsilon}(t) \geq$ 0 and $\int_{-\infty}^{\infty} \rho_{\varepsilon}(t) d t=1$. Then $g_{\varepsilon}=\rho_{\varepsilon} * g \rightarrow g$ as $\varepsilon \rightarrow 0$ in $L^{1}(J, X)$ and $v_{\varepsilon}=\rho_{\varepsilon} * v$ are solutions of (5) with $g$ replaced by $g_{\varepsilon}, v_{\varepsilon} \rightarrow v$ as $\varepsilon \rightarrow 0$ in $C(J, X)$. Since $g_{\varepsilon}$ is smooth, $A v_{\varepsilon} \in C(J, X)$ and $A v_{\varepsilon}$ is a Cauchy sequence in $L^{1}(J, X)$ as the following 
estimate shows

$$
\begin{aligned}
\mid A v_{\varepsilon}- & \left.A v_{\varepsilon^{\prime}}\right|_{L^{1}} \leq \int_{0}^{a}\left|\int_{0}^{t} A T(\tau)\left(g_{\varepsilon}(t-\tau)-g_{\varepsilon^{\prime}}(t-\tau)\right) d \tau\right| d t \\
\leq & \int_{0}^{a}\left|\int_{0}^{t} A T(\tau)\left[g_{\varepsilon}(t-\tau)-g_{\varepsilon^{\prime}}(t-\tau)-g_{\varepsilon}(t)+g_{\varepsilon^{\prime}}(t)\right] d \tau\right| d t \\
& +\int_{0}^{a}\left|(T(t)-I)\left(g_{\varepsilon}(t)-g_{\varepsilon^{\prime}}(t)\right)\right| d t \\
\leq & M \int_{0}^{a} \tau^{-1} \int_{\tau}^{a}\left|g_{\varepsilon}(t-\tau)-g_{\varepsilon^{\prime}}(t-\tau)-g_{\varepsilon}(t)+g_{\varepsilon^{\prime}}(t)\right| d t d \tau \\
& +(M+1)\left|g_{\varepsilon}-g_{\varepsilon^{\prime}}\right|_{L^{1}} \\
\leq & 2 M\left|g_{\varepsilon}-g_{\varepsilon^{\prime}}\right|_{L^{1}}^{1 / 2} \int_{0}^{a} \tau^{-1}\left(\int_{0}^{a-\tau}\left|g_{\varepsilon}(t-\tau)-g_{\varepsilon}(t)\right|\right. \\
& \left.+(M+1)\left|g_{\varepsilon}-g_{\varepsilon^{\prime}}\right|_{L^{1}} \quad+\left|g_{\varepsilon^{\prime}}(t-\tau)-g_{\varepsilon^{\prime}}(t)\right| d t\right)^{1 / 2} d \tau \\
\leq & 4 M\left|g_{\varepsilon}-g_{\varepsilon^{\prime}}\right|_{L^{1}}^{1 / 2}\left[\left(\int_{0}^{a} \tau^{\alpha / 2-1} d \tau\right)[g]_{\alpha}^{1 / 2}+2|g|_{L^{1}}^{1 / 2}\right] \rightarrow 0
\end{aligned}
$$

as $\varepsilon \rightarrow 0$; note that $\left[g_{\varepsilon}\right]_{\alpha} \leq[g]_{\alpha}$ holds. Thus $v_{\varepsilon} \rightarrow v$ and $A v_{\varepsilon} \rightarrow w$ in $L^{1}(J, X)$, hence $w=A v \in L^{1}(J, X)$, since $A$ is closed.

3. It remains to derive the estimate for $\left[A v_{\varepsilon}\right]_{\alpha}$ uniformly w.r.t. $\varepsilon>0$; we shall drop the index $\varepsilon$ in the sequel

$$
\begin{aligned}
\int_{0}^{a} \log \left(1+\frac{h}{t}\right)|A v(t)| d t \leq & \int_{0}^{a} \log \left(1+\frac{h}{t}\right)\left|\int_{0}^{t} A T(\tau)(g(t-\tau)-g(t)) d \tau\right| d t \\
& +\int_{0}^{a} \log \left(1+\frac{h}{t}\right)|(T(t)-I) g(t)| d t \\
= & I_{1}+I_{2}
\end{aligned}
$$

since $\left[g_{\varepsilon}\right]_{\alpha} \leq[g]_{\alpha}$ for all $\varepsilon>0$ this implies the first part of the desired estimate. 
To derive the second part, we decompose according to

$$
\begin{aligned}
A v(t+h)-A v(t)= & (T(t+h)-T(t)) g(t)+(T(h)-I)(g(t+h)-g(t)) \\
& +\int_{0}^{h} A T(\tau)(g(t+h-\tau)-g(t+h)) d \tau \\
& +\int_{0}^{t} A(T(h+\tau)-T(\tau))(g(t-\tau)-g(t)) d \tau \\
= & I_{1}+I_{2}+I_{3}+I_{4} .
\end{aligned}
$$

Estimation of the first term yields

$$
\int_{0}^{a-h}\left|I_{1}(t)\right| d t \leq M \int_{0}^{a} \log \left(1+\frac{h}{t}\right)|g(t)| d t \leq M[g]_{\alpha} h^{\alpha}
$$

similarly we obtain for the second one

$$
\int_{0}^{a-h}\left|I_{2}(t)\right| d t \leq(M+1) \int_{0}^{a}|g(t+h)-g(t)| d t \leq(M+1)[g]_{\alpha} h^{\alpha}
$$

for the third term we get

$$
\begin{aligned}
\int_{0}^{a-h}\left|I_{3}(t)\right| d t & \leq M \int_{0}^{h} \tau^{-1} \int_{0}^{a-h}|g(t+h-\tau)-g(t+h)| d t d \tau \\
& \leq M \int_{0}^{h} \tau^{-1} \int_{0}^{a-\tau}|g(t)-g(t+\tau)| d t d \tau \leq M[g]_{\alpha} \int_{0}^{h} \tau^{\alpha-1} d \tau \\
& =M[g]_{\alpha} \alpha^{-1} h^{\alpha}
\end{aligned}
$$

and finally

$$
\begin{aligned}
& \int_{0}^{a-h}\left|I_{4}(t)\right| d t \leq \int_{0}^{a-h} \frac{4 M^{2} h}{(\tau+h) \tau} \cdot \int_{\tau}^{a-h}|g(t-\tau)-g(t)| d t d \tau \\
& \quad \leq 4 M^{2}[g]_{\alpha} \int_{0}^{a-h} \frac{h \tau^{\alpha}}{\tau(\tau+h)} d \tau \leq 4 M^{2}[g]_{\alpha} h^{\alpha} \int_{0}^{\infty} s^{\alpha-1}(1+s)^{-1} d s \\
& \quad=4 M^{2} \pi(\sin \alpha \pi)^{-1}[g]_{\alpha} h^{\alpha}
\end{aligned}
$$

since

$$
|A(T(\tau+h)-T(\tau))|=\left|\int_{\tau}^{\tau+h} A^{2} T(s) d s\right| \leq 4 M^{2} \int_{\tau}^{\tau+h} s^{-2} d s=4 M^{2} \frac{h}{\tau(\tau+h)} .
$$

We now turn to the proofs of Theorems 2 and 3. If we replace $u(t)$ by $u_{\omega}(t)=$ $u(t) e^{-\omega t}$ in (1), $u_{\omega}$ again satisfies an equation of type (1), where $A$ has to be replaced by $A_{\omega}=A-\omega, f$ by $f_{\omega}(t)=f(t) e^{-\omega t}$ and $B(t)$ by $B_{\omega}(t)=\int_{0}^{t} d B(\tau) e^{-\omega \tau}$. With $T_{\omega}(t)=T(t) e^{-\omega t}$ we obtain $\left|T_{\omega}(t)\right| \leq|T(t)| \leq M$ and

$$
\left|A_{\omega} T_{\omega}(t)\right| \leq|A T(t)|+t^{-1}|T(t)|\left(\omega t e^{-\omega t}\right) \leq M t^{-1}\left(1+e^{-1}\right),
$$

the crucial estimates (6) hold uniformly for $\omega \geq 0$. On the other hand, choosing $\omega$ large enough, the variation of $B_{\omega}(t)$ can be made arbitrarily small, by virtue of

$$
\left.\operatorname{Var} B_{\omega}\right|_{0} ^{a} \leq \int_{0}^{a}|d B(\tau)|_{Y, X} \leq \int_{0}^{a} d b(\tau) e^{-\omega \tau} \leq b(\varepsilon)+(b(a)-b(\varepsilon)) e^{-\omega \varepsilon}
$$


and since $b(\varepsilon) \rightarrow 0$ as $\varepsilon \rightarrow 0+$. Therefore, we may assume $\left.\operatorname{Var} B\right|_{0} ^{a}=b(a) \leq \eta$ in the sequel, where $\eta$ is as small as we need.

Proof of TheOREM 2. Let $u_{0}=0$ and $f \in C^{\alpha}(J, X), f(0) \in Y_{\alpha}$ or $f \in$ $C\left(J, Y_{\alpha}\right)$. We consider the Neumann-series

$$
u=\sum_{n=0}^{\infty}(T * d B)^{* n} * T * f
$$

and show that it defines a solution of (1). Lemma 1 or Lemma 2, respectively, show that $T * f$ belongs to $C^{\alpha}(J, Y)$ and $|T * f|_{1, \alpha} \leq C(\alpha, f)$ for some constant $C(\alpha, f)$. For any function $g \in C^{\alpha}(J, Y)$ with $g(0)=0$ we obtain

$$
|d B * g|_{0,0} \leq b(a)|g|_{1,0}
$$

as well as

$$
\begin{aligned}
|(d B * g)(t+h)-(d B * g)(t)| \leq & \int_{t}^{t+h} d b(\tau)|g(t+h-\tau)|_{Y} \\
& +\int_{0}^{t} d b(\tau)|g(t+h-\tau)-g(t-\tau)|_{Y} \\
\leq & b(a) h^{\alpha} \cdot \sup _{t, h}|g(t+h)-g(t)| h^{-\alpha}
\end{aligned}
$$

hence $d B * g \in C^{\alpha}(J, Y)$ and

$$
|d B * g|_{0, \alpha} \leq b(a)|g|_{1, \alpha} \leq \eta|g|_{1, \alpha} .
$$

Since $(d B * g)(0)=0$, another application of Lemma 1 yields $T *(d B * g) \in C^{\alpha}(J, Y)$ and

$$
|T *(d B * g)|_{1, \alpha} \leq C(a, \alpha) \eta|g|_{1, \alpha} .
$$

Therefore we see inductively that each term of the series (10) is well defined, belongs to $C^{\alpha}(J, Y)$ and we have

$$
|u|_{1, \alpha} \leq \sum_{n=0}^{\infty}(C(a, \alpha) \eta)^{n} \cdot C(\alpha, f)=C(\alpha, f)(1-\eta C(a, \alpha))^{-1}
$$

provided $\eta$ is sufficiently small. It is now obvious that $u$ is a solution of (1) and that $u \in C^{1}(J, X)$ holds.

Proof of TheOREM 3. Let $u_{0} \in Y_{\alpha}$ and $f \in B^{\alpha}(J, X)$ for some $\alpha \in(0,1)$. The solution $u$ of (1) will be given by means of

$$
u=\sum_{n=0}^{\infty}(T * d B)^{* n} *\left(T u_{0}+T * f\right) .
$$

Lemma 3 shows that $g=T u_{0}+T * f$ belongs to $B^{\alpha}(J, Y)$, and that $[g]_{Y, \alpha} \leq$ $C(a, \alpha)\left(\left|u_{0}\right|_{\alpha}+[f]_{\alpha}\right)$ for some constant $C(a, \alpha)$ holds. Let $\rho_{\varepsilon}$ be as in the proof of Lemma 3 ; then $\rho_{\varepsilon} * g=g_{\varepsilon} \in C(J, Y)$ and $g_{\varepsilon} \rightarrow g$ in $L^{1}(J, Y)$ as $\varepsilon \rightarrow 0$. Since

$$
\begin{aligned}
\left|d B * g_{\varepsilon}-d B * g_{\varepsilon^{\prime}}\right|_{L^{1}} & \leq \int_{0}^{a} d b(\tau) \int_{\tau}^{a}\left|g_{\varepsilon}(t-\tau)-g_{\varepsilon^{\prime}}(t-\tau)\right|_{\hat{Y}} d t \\
& \leq b(a)\left|g_{\varepsilon}-g_{\varepsilon^{\prime}}\right|_{L^{1}}
\end{aligned}
$$


we see that $d B * g_{\varepsilon} \rightarrow w$ in $L^{1}(J, X)$ as $\varepsilon \rightarrow 0$, and therefore it makes sense to define

$$
d B * g=\lim _{\varepsilon \rightarrow 0} d B * g_{\varepsilon} \quad \text { in } L^{1}(J, X)
$$

and this does not depend on the choice of the $\rho_{\varepsilon}$. Next we show that $d B * g \in$ $B^{\alpha}(J, X)$ and $[d B * g]_{\alpha} \leq c b(a)[g]_{Y, \alpha}$. In fact, we have

$$
\begin{gathered}
\int_{0}^{a} \log \left(1+\frac{h}{t}\right)\left|d B * g_{\varepsilon}(t)\right| d t \leq \int_{0}^{a} d b(\tau) \int_{\tau}^{a} \log \left(1+\frac{h}{t}\right)\left|g_{\varepsilon}(t-\tau)\right|_{Y} d t \\
\leq \int_{0}^{a} d b(\tau) \int_{0}^{a} \log \left(1+\frac{h}{t}\right)\left|g_{\varepsilon}(t)\right|_{Y} d t \leq b(a)[g]_{Y, \alpha} h^{\alpha},
\end{gathered}
$$

and

$$
\begin{aligned}
\int_{0}^{a-h} & \left|\left(d B * g_{\varepsilon}\right)(t+h)-d B * g_{\varepsilon}(t)\right| d t \leq \int_{0}^{a-h} \int_{t}^{t+h} d b(\tau)\left|g_{\varepsilon}(t+h-\tau)\right|_{Y} d t \\
& +\int_{0}^{a-h} \int_{0}^{t} d b(\tau)\left|g_{\varepsilon}(t+h-\tau)-g_{\varepsilon}(t-\tau)\right|_{Y} d t \\
\leq & \int_{0}^{h}\left|g_{\varepsilon}(\tau)\right|_{Y} d \tau \cdot b(a)+b(a) \int_{0}^{a-h}\left|g_{\varepsilon}(t+h)-g_{\varepsilon}(t)\right|_{Y} d t \\
\leq & b(a) \int_{0}^{a} \log \left(1+\frac{h}{t}\right)|g(t)|_{Y} d t \cdot(\log 2)^{-1} \\
& +b(a) \int_{0}^{a-h}\left|g_{\varepsilon}(t+h)-g_{\varepsilon}(t)\right|_{Y} d t \\
\leq & b(a) h^{\alpha}[g]_{Y, \alpha}(\log 2)^{-1} .
\end{aligned}
$$

Another application of Lemma 3 therefore yields $T *(d B * g) \in B^{\alpha}(J, Y)$ and $[T *(d B * g)]_{Y, \alpha} \leq C(a, \alpha) b(a)[g]_{Y, \alpha}$. Thus, by induction each term in the series (11) is well defined, and

$$
\begin{aligned}
{[u]_{Y, \alpha} } & \leq \sum_{n=0}^{\infty}(C(a, \alpha) b(a))^{n} \cdot C(a, \alpha)\left(\left|u_{0}\right|_{\alpha}+[f]_{\alpha}\right) \\
& \leq(1-C(a, \alpha) b(a))^{-1} C(a, \alpha)\left(\left|u_{0}\right|_{\alpha}+[f]_{\alpha}\right)<\infty
\end{aligned}
$$

provided that $b(a)$ is sufficiently small. The assertions now follow easily by means of another approximation argument. In fact if $T u_{0}+T * f$ in (11) is replaced by $T *\left(\rho_{\varepsilon} u_{0}+\rho_{\varepsilon} * f\right)$, then the resulting $u_{\varepsilon}$ is a solution of (1) by Theorem 2 and we have $u_{\varepsilon} \rightarrow u$ in $L^{1}(J, Y)$, hence also $u_{\varepsilon} \rightarrow u$ in $W^{1,1}(J, X)$ as equation (1) shows.

4. PROOF OF THEOREMS 1 AND 4. To introduce some simplifications, note first that we may assume $B \in \mathrm{BV}\left(\mathbf{R}_{+}, \mathbf{B}(Y, X)\right)$ since the resolvent $S(t)$ is determined by $A$ and the values of $B(s)$ for $s \leq t$ only. Next we may assume that $A$ is invertible and that the variation of $B$ is as small as we please, say $\left.\operatorname{Var} B\right|_{0} ^{\infty}=b(\infty) \leq \eta_{0}$; this follows from the remarks in front of the proof of Theorem 2. Finally define $K(t)=B(t) A^{-1}$; obviously we have $K \in \mathrm{BV}\left(\mathbf{R}_{+}, \mathbf{B}(X)\right)$ and $\left.\operatorname{Var} K\right|_{0} ^{\infty} \leq b(\infty) \leq \eta_{0}$, since the norm on $Y$ may be chosen as $|x|_{Y}=|A x|$ now. 
Formally, the resolvent $S(t)$ and its similarity transform $S_{1}(t)=A S(t) A^{-1}$ are given by the Neumann series

$$
\begin{gathered}
S=T+T * d K * A t+T * d K * A T * d K * A T+\cdots \quad \text { and } \\
S_{1}=T+A T * d K * T+A T * d K * A T * d K * T+\cdots .
\end{gathered}
$$

In the first step of the proof we show by the arguments in the proof of Theorem 3 that both series make sense in $L^{1}\left(\mathbf{R}_{+}, \mathbf{B}(X)\right)$, and that $S \in W^{1,1}\left(\mathbf{R}_{+}, \mathbf{B}(Y, X)\right)$ holds. Since $A S(t) A^{-1}=S_{1}(t)$ is also satisfied we therefore obtain $S \in$ $L^{1}\left(\mathbf{R}_{+}, \mathbf{B}(Y)\right)$ and that the resolvent equations (3) hold in $L^{1}\left(\mathbf{R}_{+}, \mathbf{B}(Y, X)\right)$.

The second step consists in proving the boundedness of $S(t)$ and $S_{1}(t)$ in $\mathbf{B}(X)$, and that of $S^{\prime}(t)$ in $\mathbf{B}(Y, X)$. This is the most difficult part of the proof, since now pointwise estimates are needed. These will be obtained by a term by term estimation of (12) and are based on the following inequalities.

$$
\begin{array}{ll}
|T(t)| \leq M e^{-t} \quad \text { and } \quad|A T(t)| \leq \frac{M e^{-t}}{t} & \text { for } t>0, \\
|T(t)-T(s)| \leq M e^{-s} \log \left(1+\frac{t-s}{s}\right) & \text { and } \\
|A(T(t)-T(s))| \leq M e^{-s} \cdot \frac{t-s}{t s} & \text { for } t>s>0 .
\end{array}
$$

The third part of the proof then is routine; by standard approximation and density arguments we show that $S(t)$ and $S_{1}(t)$ are strongly continuous.

At several places we shall take advantage of Laplace transform theory; standard references are Widder [19] for scalar functions and Hille and Phillips $[\mathbf{1 2}]$ for vectorvalued functions. Recall that for $f \in L^{1}\left(\mathbf{R}_{+}, X\right)$ its Laplace transform is defined by

$$
\hat{f}(\lambda)=\int_{0}^{\infty} e^{-\lambda t} f(t) d t, \quad \operatorname{Re} \lambda \geq 0
$$

and for $g \in \mathrm{BV}\left(\mathbf{R}_{+}, X\right)$ we let

$$
\widehat{d g}(\lambda)=\int_{0}^{\infty} e^{-\lambda t} d g(t), \quad \operatorname{Re} \lambda \geq 0 .
$$

Note that $\widehat{d g}(\lambda)=\lambda \hat{g}(\lambda)$ holds and recall also the convolution theorem

$$
\widehat{d K * f(\lambda)}=\widehat{d K}(\lambda) \hat{f}(\lambda), \quad \operatorname{Re} \lambda \geq 0 .
$$

Finally the uniqueness theorem for the Laplace transform states that if $f_{1}, f_{2} \in$ $L^{1}\left(\mathbf{R}_{+}, X\right)$ are such that $\hat{f}_{1}(\lambda) \equiv \hat{f}_{2}(\lambda)$ then $f_{1}=f_{2}$ in $L^{1}\left(\mathbf{R}_{+}, X\right)$.

STEP 1. Let $H(\lambda)=(\lambda-A-\widehat{d B}(\lambda))^{-1}$ for $\operatorname{Re} \lambda \geq 0$. Then there is $S \in$ $L^{1}\left(\mathbf{R}_{+}, \mathbf{B}(X)\right) \cap L^{1}\left(\mathbf{R}_{+}, \mathbf{B}(Y)\right) \cap W^{1,1}\left(\mathbf{R}_{+}, \mathbf{B}(Y, X)\right)$ such that $\hat{S}(\lambda)=H(\lambda)$ for $\operatorname{Re} \lambda \geq 0$. In particular, $S$ satisfies the resolvent equations $(3)$ in $L^{\mathbf{1}}\left(\mathbf{R}_{+}, \mathbf{B}(Y, X)\right)$.

ProOF. Note that $\lambda-A-\widehat{d B}(\lambda)=\left(I-\widehat{d K}(\lambda) A(\lambda-A)^{-1}\right)(\lambda-A)$ is invertible for each $\lambda$ with $\operatorname{Re} \lambda \geq 0$ since

$$
\left|\widehat{d K}(\lambda) A(\lambda-A)^{-1}\right| \leq \widehat{d K}(\lambda)|| A(\lambda-A)^{-1} \mid \leq \eta_{0} \cdot C<1
$$

holds for $\operatorname{Re} \lambda \geq 0$. Thus we obtain

$$
\begin{aligned}
H(\lambda) & =(\lambda-A)^{-1}\left(I-\widehat{d K}(\lambda) A(\lambda-A)^{-1}\right)^{-1} \\
& =(\lambda-A)^{-1}+(\lambda-A)^{-1} \widehat{d K}(\lambda) A(\lambda-A)^{-1}+\cdots
\end{aligned}
$$


and for $H_{1}(\lambda)=A H(\lambda) A^{-1}$ we have

$$
H_{1}(\lambda)=(\lambda-A)^{-1}+A(\lambda-A)^{-1} \widehat{d K}(\lambda)(\lambda-A)^{-1}+\cdots
$$

i.e. $H(\lambda)$ and $H_{1}(\lambda)$ are well defined and are the formal Laplace transforms of (12).

By means of (13) we see that $T \in B^{\alpha}\left(\mathbf{R}_{+}, \mathbf{B}(X)\right)$ for each $\alpha \in(0,1)$; in fact

$$
\int_{0}^{\infty} \log \left(1+\frac{h}{t}\right)|T(t)| d t \leq M \alpha^{-1} h^{\alpha} \int_{0}^{\infty} t^{-\alpha} e^{-t} d t=\Gamma(1-\alpha) \alpha^{-1} M h^{\alpha}
$$

and

$$
\int_{0}^{\infty}|T(t+h)-T(t)| d t \leq M \int_{0}^{\infty} e^{-t} \log \left(1+\frac{h}{t}\right) d t \leq \Gamma(1-\alpha) \alpha^{-1} M h^{\alpha}
$$

where $\Gamma(\beta)$ denotes the gamma function. Here we have used the elementary inequality

$$
\frac{x}{1+x} \leq \log (1+x) \leq \alpha^{-1} x^{\alpha} \quad \text { for all } x>0, \alpha \in(0,1]
$$

which will frequently be applied, in the sequel. As in the proof of Theorem 3 we then obtain $d K * T \in B^{\alpha}\left(\mathbf{R}_{+}, \mathbf{B}(X)\right)$ and

$$
[d K * T]_{\alpha} \leq 2(\log 2)^{-1} \eta_{0}[T]_{\alpha}
$$

Next, the arguments in the proof of Lemma 3 show that $A T *(d K * T) \in$ ${ }^{\alpha}\left(\mathbf{R}_{+}, \mathbf{B}(X)\right)$ and

$$
[A T *(d K * T)]_{\alpha} \leq C(\alpha \sin \alpha \pi)^{-1}[d K * T]_{\alpha} \leq C(\alpha) \eta_{0}[T]_{\alpha}
$$

as well as $T *(d K * T) \in B^{1+\alpha}\left(\mathbf{R}_{+}, \mathbf{B}(X)\right)$ and

$$
\left[\frac{d}{d t} T *(d K * T)\right]_{\alpha} \leq C(\alpha) \eta_{0}[T]_{\alpha} .
$$

By induction, each term of the series (12) for $S_{1}(t)$ is therefore well defined and belongs to $B^{\alpha}\left(\mathbf{R}_{+}, \mathbf{B}(X)\right)$; the series is convergent in $B^{\alpha}\left(\mathbf{R}_{+}, \mathbf{B}(X)\right)$ since

$$
\left[S_{1}\right]_{\alpha} \leq \sum_{n=0}^{\infty}\left(C(\alpha) \eta_{0}\right)^{n}[T]_{\alpha}=\left(1-C(\alpha) \eta_{0}\right)^{-1}[T]_{\alpha}
$$

provided $\eta_{0}$ is small enough. Also, $A^{-1} S_{1}$ exists in $B^{1+\alpha}\left(\mathbf{R}_{+}, \mathbf{B}(X)\right)$ as the second estimate shows. B Similarly, we obtain $S \in B^{\alpha}\left(\mathbf{R}_{+}, \mathbf{B}(X)\right)$ be means of the series (12) for $S(t)$, where we are now working from the left to the right. Taking Laplace transform, equations (12) and (14) yield

$$
\hat{S}(\lambda)=H(\lambda) \quad \text { and } \quad \hat{S}_{1}(\lambda)=H_{1}(\lambda), \quad \operatorname{Re} \lambda \geq 0,
$$

and since $H_{1}(\lambda)=A H(\lambda) A^{-1}$ holds, uniqueness of the Laplace transform implies $S_{1}(t)=A S(t) A^{-1}$ for a.a. $t \geq 0$. Thus $S \in L^{1}\left(\mathbf{R}_{+}, \mathbf{B}(X)\right) \cap L^{1}\left(\mathbf{R}_{+}, \mathbf{B}(Y)\right) \cap$ $W^{1,1}\left(\mathbf{R}_{+}, \mathbf{B}(Y, X)\right)$ and each term in the resolvent equations makes sense in $L^{1}\left(\mathbf{R}_{+}, \mathbf{B}(Y, X)\right)$. By means of $\hat{S}(\lambda)=H(\lambda)=(\lambda-A-\widehat{d B}(\lambda))^{-1}$ we finally get

$$
\begin{aligned}
\hat{S}^{\prime}(\lambda) & =\lambda \hat{S}(\lambda)-I=(A+\widehat{d B}(\lambda)) \hat{S}(\lambda)=\widehat{A S}(\lambda)+\widehat{d B * S}(\lambda) \\
& =\hat{S}(\lambda)(A+\widehat{d B}(\lambda))=\widehat{S A}(\lambda)+\widehat{S * d B}(\lambda),
\end{aligned}
$$


hence (3) holds in $L^{1}\left(\mathbf{R}_{+}, \mathbf{B}(Y, X)\right)$, by uniqueness of the Laplace transform again.

STEP 2. The resolvent $S(t)$ defined in Step 1 satisfies

$$
S \in L^{\infty}\left(\mathbf{R}_{+}, \mathbf{B}(X)\right) \cap L^{\infty}\left(\mathbf{R}_{+}, \mathbf{B}(Y)\right) \cap W^{1, \infty}\left(\mathbf{R}_{+}, \mathbf{B}(Y, X)\right) .
$$

Proof. (a) Let $\varepsilon>0$ and let $\rho_{\varepsilon} \in C_{0}^{\infty}$ be such that $\rho_{\varepsilon}(t) \geq 0$, supp $\rho_{\varepsilon} \subset(0, \varepsilon)$ and $\int_{-\infty}^{\infty} \rho_{\varepsilon}(t) d t=1$. Define approximate kernels $K_{\varepsilon}(t)$ be means of

$$
K_{\varepsilon}(t)=(I-\varepsilon A)^{-1}\left(\rho_{\varepsilon} * d K\right)(t),
$$

and let $S_{\varepsilon}(t)$ denote the resolvent of

$$
u^{\prime}=A u+K_{\varepsilon} * A u+f
$$

i.e.

$$
\begin{aligned}
S_{\varepsilon} & =T * \sum_{n=0}^{\infty}\left(K_{\varepsilon} * A T\right)^{* n} \quad \text { and } \\
S_{1 \varepsilon} & =A S_{\varepsilon} A^{-1}=\sum_{n=0}^{\infty}\left(A T * K_{\varepsilon}\right)^{* n} * T .
\end{aligned}
$$

Since $K_{\varepsilon}$ is a smooth $Y$-valued kernel, the series are well defined, the integrands of the summands have no singularities, everything is continuous. For this reason we shall derive uniform estimates for $S_{1 \varepsilon}$ first. Note that $\left|K_{\varepsilon}\right|_{L^{1}} \leq C_{0}|d K|_{1} \leq C_{0} \eta_{0}=$ $\eta$ holds.

(b) Claim 1. Let

$$
R_{n}(t)=\left(A T * K_{\varepsilon}\right)^{* n} * T(t) \quad \text { for } t \geq 0, n \in N
$$

denote the $n$th summand of the series (17) for $S_{1 \varepsilon}$. Then

$$
\begin{aligned}
& R_{n}(t)=\int_{0}^{t} \int_{0}^{t_{n}} \cdots \int_{0}^{t_{2}} \int_{\tau}^{t} \int_{\tau}^{s_{n}} \\
& \cdots \int_{\tau}^{s_{2}} A T\left(t-s_{n}\right) K_{\varepsilon}\left(\tau_{n}\right) A T\left(s_{n}-s_{n-1}\right) K_{\varepsilon}\left(\tau_{n-1}\right) \\
& \cdots K_{\varepsilon}\left(\tau_{1}\right) T\left(s_{1}-\tau\right) d s_{1} \cdots d s_{n} d \tau_{1} \cdots d \tau_{n}
\end{aligned}
$$

holds, where $t_{k}=t-\sum_{k}^{n} \tau_{j}$ and $\tau=\sum_{1}^{n} \tau_{j}$.

PROOF. Since the integrand is continuous, we may change the order of integration as we please. The induction step from $n$ to $n+1$ is verified as follows.

$$
\begin{aligned}
R_{n+1}(t)= & A T * K_{\varepsilon} * R_{n}(t)=\int_{0}^{t} A T(t-s) \int_{0}^{s} K_{\varepsilon}(\rho) R_{n}(s-\rho) d \rho d s \\
= & \int_{0}^{t} \int_{\rho}^{t} A T(t-s) K_{\varepsilon}(\rho) R_{n}(s-\rho) d s d \rho \\
= & \int_{0}^{t} \int_{\rho}^{t} \int_{0}^{s-\rho} \int_{0}^{s-\rho-\tau_{n}} \\
& \cdots \int_{0}^{s-\rho-\sum_{2}^{n} \tau_{j}} \int_{0}^{s-\rho} \int_{\tau}^{s_{n}} \int_{\tau}^{s_{2}} A T(t-s) K_{\varepsilon}(\rho) A T\left(s-\rho-s_{n}\right) \\
& \cdots K_{\varepsilon}\left(\tau_{1}\right) T\left(s_{1}-\tau\right) d s_{1} d s_{2} \cdots d s_{n} d \tau_{1} \cdots d \tau_{n} d s d \rho .
\end{aligned}
$$


The transformation $s_{j} \rightarrow s_{j}+\rho$ and $\tau^{\prime}=\tau+\rho$ yields

$$
\begin{array}{r}
R_{n+1}(t)=\int_{0}^{t} \int_{\rho}^{t} \int_{0}^{s-\rho} \cdots \int_{0}^{s-\rho-\sum_{2}^{n} \tau_{j}} \int_{\tau^{\prime}}^{s} \int_{\tau^{\prime}}^{s_{n}} \int_{\tau^{\prime}}^{s_{2}} A T(t-s) K_{\varepsilon}(\rho) A T\left(s-s_{n}\right) \\
\cdots K_{\varepsilon}\left(\tau_{1}\right) T\left(s_{1}-\tau^{\prime}\right) d s_{1} \cdots d s_{n} d \tau_{1} \cdots d \tau_{n} d s d \rho
\end{array}
$$

and commuting the integral over $s$ with those over $\tau_{1} \cdots \tau_{n}$ leads to

$$
R_{n+1}(t)=\int_{0}^{t} \int_{0}^{t_{n+1}} \cdots \int_{0}^{t_{2}} \int_{\tau^{\prime}}^{t} \int_{\tau^{\prime}}^{s_{n}} \cdots \int_{\tau^{\prime}}^{s_{2}}\{\cdots\} d s_{1} \cdots d s_{n} d s d \tau_{1} \cdots d \tau_{n} d \rho
$$

where $t_{j}=t-\rho-\sum_{j}^{n} \tau_{k}$, i.e. (19) holds for $n+1$.

The representation (19) of $R_{n}(t)$ suggests to consider

$$
Z_{n}(t)=\int_{\tau}^{t} \int_{\tau}^{s_{n}} \cdots \int_{\tau}^{s_{2}} A T\left(t-s_{n}\right) K_{\varepsilon}\left(\tau_{n}\right) \cdots K_{\varepsilon}\left(\tau_{1}\right) T\left(s_{1}-\tau\right) d s_{1} \cdots d s_{n}
$$

first, where the dependence on $\tau_{1}, \ldots, \tau_{n}$ has been dropped. To obtain the desired estimates we decompose $Z_{n}(t)$ according to

Claim 2. We have

$$
\begin{aligned}
Z_{n}(t)= & \int_{\tau}^{t} A T(t-s) U_{n}(t, s) d s \\
& +\int_{\tau}^{t}(T(t-s)-I) V_{n}(t, s) d s+(T(t-\tau)-I) W_{n}(t),
\end{aligned}
$$

where $U_{n}, V_{n}, W_{n}$ are defined inductively by

$$
\begin{aligned}
& U_{1}(t, s)=K_{\varepsilon}\left(\tau_{1}\right)(T(s-\tau)-T(t-\tau)), \\
& V_{1}(t, s) \equiv 0, \quad W_{1}(t)=K_{\varepsilon}\left(\tau_{1}\right) T(t-\tau)
\end{aligned}
$$

and

$$
W_{n+1}(t)=K_{\varepsilon}\left(\tau_{n+1}\right)(T(t-\tau)-I) W_{n}(t),
$$

$$
\left.V_{n+1}(t, s)=K_{\varepsilon}\left(\tau_{n+1}\right)\left[A T(t-s) U_{n}(t, s)+(T(t-s)-I) V_{n} \quad s\right)\right],
$$

$$
\begin{aligned}
U_{n+1}(t, s)=K_{\varepsilon}\left(\tau_{n+1}\right)\{ & (T(s-\tau)-I) W_{n}(s)-(T(t-\tau)-I) W_{n}(t) \\
& +\int_{\tau}^{s}\left(A T(s-r) U_{n}(s, r)-A T(t-r) U_{n}(t, r)\right) d r \\
& \left.+\int_{\tau}^{s}\left[(T(s-r)-I) V_{n}(s, r)-(T(t-r)-I) V_{n}(t, r)\right] d r\right\} .
\end{aligned}
$$

Proof. For $n=1$ we have

$$
\begin{aligned}
Z_{1}(t)= & \int_{\tau}^{t} A T(t-s) K_{\varepsilon}\left(\tau_{1}\right) T(s-\tau) d s \\
= & \int_{\tau}^{t} A T(t-s) K_{\varepsilon}\left(\tau_{1}\right)(T(s-\tau)-T(t-\tau)) d s \\
& +\int_{\tau}^{t} A T(t-s) d s K_{\varepsilon}\left(\tau_{1}\right) T(t-\tau)
\end{aligned}
$$


hence $(20),(21)$ holds for $n=1$. Let the assertions be true for $n$; then we have

$$
\begin{aligned}
Z_{n+1}( & t)=\int_{\tau}^{t} A T(t-s) K_{\varepsilon}\left(\tau_{n+1}\right) Z_{n}(s) d s \\
= & \int_{\tau}^{t} A T(t-s) K_{\varepsilon}\left(\tau_{n+1}\right) \int_{\tau}^{s} A T(s-r) U_{n}(s, r) d r d s \\
& +\int_{\tau}^{t} A T(t-s) K_{\varepsilon}\left(\tau_{n+1}\right) \int_{\tau}^{s}(T(s-r)-I) V_{n}(s, r) d r d s \\
& +\int_{\tau}^{t} A T(t-s) K_{\varepsilon}\left(\tau_{n+1}\right)(T(s-\tau)-I) W_{n}(s) d s \\
= & \int_{\tau}^{t} A T(t-s) K_{\varepsilon}\left(\tau_{n+1}\right)\left\{\int_{\tau}^{s}\left[A T(s-r) U_{n}(s, r)-A T(t-r) U_{n}(t, r)\right] d r\right. \\
\quad & \quad+\int_{\tau}^{s}\left[(T(s-r)-I) V_{n}(s, r)-(T(t-r)-I) V_{n}(t, r)\right] d r \\
& +\int_{\tau}^{t} A T(t-s) K_{\varepsilon}\left(\tau_{n+1}\right) \\
& +\left\{\int_{\tau}^{s}\left[A T(t-r) U_{n}(t, r)+(T(t-r)-I) V_{n}(t, r)\right] d r+(T(t-\tau)-I) W_{n}(t)\right\} d s \\
= & \int_{\tau}^{t} A T(t-s) U_{n+1}(t, s) d s+\int_{\tau}^{t}(T(t-r)-I) V_{n+1}(t, r) d r
\end{aligned}
$$

hence the assertion also holds for $n+1$.

(c) To obtain the desired estimate for $Z_{n}(t)$ we have to estimate $U_{n}, V_{n}, W_{n}$ separately by induction. This is easy for $W_{n}$.

Claim 3. Let $\alpha_{n}=\prod_{1}^{n}\left|K_{\varepsilon}\left(\tau_{j}\right)\right|$ and $C \geq 2 M+1$. Then

$$
\left|W_{n}(t)\right| \leq \alpha_{n} C^{n} \text { for all } t \geq 0 \text {, and }
$$

$$
\left|W_{n}(t)-W_{n}(s)\right| \leq \alpha_{n} C^{n} \varphi_{1}\left(\frac{t-s}{s-\tau}\right) \quad \text { for all } t>s>\tau
$$

where $\varphi_{1}(a)=\log (1+a)$.

PROOF. We have by (13)

$$
\left|W_{1}(t)\right| \leq\left|K_{\varepsilon}\left(\tau_{1}\right)\right| \cdot M \leq \alpha_{1} C
$$

and

$$
\left|W_{1}(t)-W_{1}(s)\right| \leq\left|K_{\varepsilon}\left(\tau_{1}\right)\right||T(t-\tau)-T(s-\tau)| \leq \alpha_{1} C \log \left(1+\frac{t-s}{s-\tau}\right),
$$

i.e. (25) and (26) for $n=1$. For the induction step we obtain with (22)

$$
\begin{aligned}
\left|W_{n+1}(t)\right| & \leq\left|K_{\varepsilon}\left(\tau_{n+1}\right)\right|(M+1)\left|W_{n}(t)\right| \\
& \leq \alpha_{n+1}(M+1) C^{n} \leq \alpha_{n+1} C^{n+1}
\end{aligned}
$$


and

$$
\begin{aligned}
\left|W_{n+1}(t)-W_{n+1}(s)\right| \leq & \left|K_{\varepsilon}\left(\tau_{n+1}\right)\right||T(t-\tau)-T(s-\tau)|\left|W_{n}(t)\right| \\
& +\left|K_{\varepsilon}\left(\tau_{n+1}\right)\right||T(s-\tau)-I|\left|W_{n}(t)-W_{n}(s)\right| \\
\leq & \left|K_{\varepsilon}\left(\tau_{n+1}\right)\right| \alpha_{n} C^{n} \log \left(1+\frac{t-s}{s-\tau}\right)(M+M+1) \\
= & \alpha_{n+1} C^{n+1} \varphi_{1}\left(\frac{t-s}{s-\tau}\right) \cdot
\end{aligned}
$$

To derive the estimates for $U_{n}$ and $V_{n}$ we define functions $\varphi_{n}(a)$ inductively by

$$
\varphi_{n+1}(a)=\int_{0}^{1} \varphi_{n}\left(\frac{1-\sigma}{\sigma}\right) \log \left(1+\frac{a}{1-\sigma}\right) \frac{d \sigma}{1-\sigma}
$$

Some properties of $\varphi_{n}$ are collected as

Claim 4. For all $n \in \mathbf{N}$ we have

(i) $0 \leq \varphi_{n}(a) \leq c(n, \beta) a^{\beta}, a \geq 0, \beta \in(0,1)$;

(ii) $\varphi_{n}(a) \leq \varphi_{n+1}(a)$ for all $a \geq 0$;

(iii) $\varphi_{n}(a)$ is increasing for all $a \geq 0$;

(iv) $\varphi_{n}(a) / a$ is decreasing for all $a \geq 0$.

ProOF. By means of (15) we obtain for the induction step

$$
\begin{aligned}
0 & \leq \varphi_{n+1}(a) \leq \int_{0}^{1} C(n, \beta+\delta)\left(\frac{1-\sigma}{\sigma}\right)^{\beta+\delta}\left(\frac{a}{1-\sigma}\right)^{\beta} \beta^{-1} \frac{d \sigma}{\sigma} \\
& =C(n, \beta+\delta) \beta^{-1} a^{\beta} \int_{0}^{1} \sigma^{-(\beta+\delta)}(1-\sigma)^{\delta-1} d \sigma \\
& \leq C(n+1, \beta) a^{\beta}
\end{aligned}
$$

where $\delta>0$ is such that $\beta+\delta<1$. Again using (15) we get

$$
\begin{aligned}
\varphi_{2}(a) & =\int_{0}^{1} \log \left(1+\frac{1-\sigma}{\sigma}\right) \log \left(1+\frac{a}{1-\sigma}\right) \frac{d \sigma}{1-\sigma} \\
& \geq \log (1+a) \int_{0}^{1} \frac{(1-\sigma) / \sigma}{1+(1-\sigma) / \sigma} \cdot \frac{d \sigma}{1-\sigma} \\
& =\log (1+a)=\varphi_{1}(a) ;
\end{aligned}
$$

hence (ii) follows inductively. (iii) and (iv) are trivial since for any $\alpha>0, \log (1+\alpha x)$ is increasing and $x^{-1} \log (1+\alpha x)$ decreasing for $x>0$. $V_{n}$.

By means of the functions $\varphi_{n}(a)$ we obtain the following estimates for $U_{n}$ and 
Claim 5. Let $C \geq 8 M+2$. Then for each $n \in \mathbf{N}$ we have

$$
\begin{aligned}
& \left|U_{n}(t, s)\right| \leq C^{n} \alpha_{n} \varphi_{n}\left(\frac{t-s}{s-\tau}\right), \quad t>s>\tau ; \\
& \left|V_{n}(t, s)\right| \leq C^{n} \alpha_{n}(t-s)^{-1} \varphi_{n-1}\left(\frac{t-s}{s-\tau}\right), \quad t>s>\tau ; \\
& \left|U_{n}(t, s)-U_{n}(\bar{t}, s)\right| \leq C^{n} \alpha_{n} \frac{t-\bar{t}}{\bar{t}-s} \cdot \varphi_{n}\left(\frac{\bar{t}-s}{s-\tau}\right), \quad t>\bar{t}>s>\tau ; \\
& \left|V_{n}(t, s)-V_{n}(\bar{t}, s)\right| \leq C^{n} \alpha_{n}(\bar{t}-s)^{-1} \varphi_{1}\left(\frac{t-\bar{t}}{\bar{t}-s}\right) \varphi_{n-1}\left(\frac{\bar{t}-s}{s-\tau}\right), \\
& t>\bar{t}>s>\tau .
\end{aligned}
$$

PRoOF. (i) For $n=1,(29)$ and (31) are trivial; with (13) we get

$$
\left|U_{1}(t, s)\right| \leq\left|K_{\varepsilon}\left(\tau_{1}\right)\right||T(s-\tau)-T(t-\tau)| \leq \alpha_{1} C \cdot \log \left(1+\frac{t-s}{s-\tau}\right),
$$

and

$$
\left|U_{1}(t, s)-U_{1}(\bar{t}, s)\right| \leq \alpha_{1}|T(t-\tau)-T(\bar{t}-\tau)| \leq \alpha_{1} \cdot C \cdot \log \left(1+\frac{t-\tau}{\bar{t}-\tau}\right),
$$

hence with (15)

$$
\log \left(1+\frac{t-\tau}{\bar{t}-\tau}\right) \leq \frac{t-\tau}{\bar{t}-\tau}=\frac{t-\bar{t}}{\bar{t}-s} \cdot \frac{(\bar{t}-s) /(s-\tau)}{1+(\bar{t}-s) /(s-\tau)} \leq \frac{t-\bar{t}}{\bar{t}-s} \log \left(1+\frac{\bar{t}-s}{s-\tau}\right)
$$

i.e. the assertion holds for $n=1$.

(ii) Let $(28) \sim(31)$ for $n$ be satisfied. Then (23) and (13) yield

$$
\begin{aligned}
& \left|V_{n+1}(t, s)\right| \leq\left|K_{\varepsilon}\left(\tau_{n+1}\right)\right| \\
& \quad \cdot\left\{M(t-s)^{-1} \alpha_{n} C^{n} \varphi_{n}\left(\frac{t-s}{s-\tau}\right)+(M+1) C^{n} \alpha_{n}(t-s)^{-1} \varphi_{n-1}\left(\frac{t-s}{s-\tau}\right)\right\} \\
& \quad \leq \alpha_{n+1} C^{n+1}(t-s)^{-1} \varphi_{n}\left(\frac{t-s}{s-\tau}\right)
\end{aligned}
$$

since $\varphi_{n-1}(a) \leq \varphi_{n}(a)$ by Claim 4(ii). Furthermore

$$
\begin{aligned}
& \left|V_{n+1}(t, s)-V_{n+1}(\bar{t}, s)\right| \\
& \leq\left|K_{\varepsilon}\left(\tau_{n+1}\right)\right|\left\{\left|(A T(t-s)-A T(\bar{t}-s)) U_{n}(\bar{t}, s)\right|+|A T(t-s)|\right. \\
& \cdot\left|U_{n}(t, s)-U_{n}(\bar{t}, s)\right|+|T(t-s)-T(\bar{t}-s)|\left|V_{n}(\bar{t}, s)\right| \\
& \left.+|T(t-s)-I|\left|V_{n}(t, s)-V_{n}(\bar{t}, s)\right|\right\} \\
& \leq \alpha_{n+1} C^{n}\left\{2 M \frac{t-\bar{t}}{(t-s)(\bar{t}-s)} \varphi_{n}\left(\frac{\bar{t}-s}{s-\tau}\right)\right. \\
& \left.+(2 M+1)(\bar{t}-s)^{-1} \varphi_{1}\left(\frac{t-\bar{t}}{\bar{t}-s}\right) \varphi_{n-1}\left(\frac{\bar{t}-s}{s-\tau}\right)\right\} \\
& \leq \alpha_{n+1} C^{n+1}(\bar{t}-s)^{-1} \varphi_{1}\left(\frac{t-\bar{t}}{\bar{t}-s}\right) \varphi_{n}\left(\frac{\bar{t}-s}{s-\tau}\right)
\end{aligned}
$$


hence (29) and (31) hold for $n+1$; here we used (15) as

$$
\begin{aligned}
\frac{t-\bar{t}}{t-s} & =\frac{t-\bar{t}}{t-\bar{t}+t-s}=\frac{(\bar{t}-t) /(\bar{t}-s)}{1+(t-\bar{t}) /(\bar{t}-s)} \\
& \leq \log \left(1+\frac{t-\bar{t}}{\bar{t}-s}\right)=\varphi_{1}\left(\frac{t-\bar{t}}{\bar{t}-s}\right)
\end{aligned}
$$

and Claim 4(ii).

(iii) For $\left|U_{n+1}(t, s)\right|$ we obtain

$$
\begin{aligned}
& \left|U_{n+1}(t, s)\right| \leq\left|K_{\varepsilon}\left(\tau_{n+1}\right)\right|\left\{|T(s-\tau)-T(t-\tau)|\left|W_{n}(s)\right|\right. \\
& +|T(t-\tau)-I|\left|W_{n}(t)-W_{n}(s)\right| \\
& +\int_{\tau}^{s}\left(|A T(s-r)-A T(t-r)|\left|U_{n}(s, r)\right|\right. \\
& \left.+|A T(t-r)|\left|U_{n}(s, r)-U_{n}(t, r)\right|\right) d r \\
& +\int_{\tau}^{s}\left(|T(s-r)-T(t-r)|\left|V_{n}(s, r)\right|\right. \\
& \left.\left.+|T(t-r)-I|\left|V_{n}(s, r)-V_{n}(t, r)\right|\right) d r\right\} \\
& \leq \alpha_{n+1} C^{n}\left\{(2 M+1) \varphi_{1}\left(\frac{t-s}{s-\tau}\right)+2 M \int_{\tau}^{s} \frac{t-s}{(s-r)(t-r)} \cdot \varphi_{n}\left(\frac{s-r}{r-\tau}\right) d r\right. \\
& \left.+(2 M+1) \int_{\tau}^{s} \varphi_{1}\left(\frac{t-s}{s-r}\right)(s-r)^{-1} \varphi_{n-1}\left(\frac{s-r}{r-\tau}\right) d \tau\right\} \\
& \leq \alpha_{n+1} C^{n+1} \cdot 2^{-1}\left\{\varphi_{1}\left(\frac{t-s}{s-\tau}\right)+\int_{\tau}^{s}(s-r)^{-1} \varphi_{1}\left(\frac{t-s}{s-r}\right) \varphi_{n}\left(\frac{s-r}{r-\tau}\right) d r\right\}
\end{aligned}
$$

since

$$
\frac{t-s}{t-r} \leq \varphi_{1}\left(\frac{t-s}{s-r}\right)
$$

by (15). The transformation

$$
r=\tau+(s-\tau) \sigma
$$

now yields

$$
\begin{aligned}
\int_{\tau}^{s}(s & -r)^{-1} \varphi_{1}\left(\frac{t-s}{s-r}\right) \varphi_{n}\left(\frac{s-r}{r-\tau}\right) d r \\
& =\int_{0}^{1} \varphi_{n}\left(\frac{1-\sigma}{\sigma}\right) \log \left(1+\frac{t-s}{s-\tau}(1-\sigma)^{-1}\right) \frac{d \sigma}{1-\sigma} \\
& =\varphi_{n+1}\left(\frac{t-s}{s-\tau}\right)
\end{aligned}
$$

hence by Claim 4(ii) we obtain (30) for $n+1$. 
(iv) Similarly we get

$$
\begin{aligned}
& \left|U_{n+1}(t, s)-U_{n}(\bar{t}, s)\right| \\
& \leq\left|K_{\varepsilon}\left(\tau_{n+1}\right)\right|\left\{|T(t-\tau)-T(\bar{t}-\tau)|\left|W_{n}(t)\right|+|T(\bar{t}-\tau)-I|\left|W_{n}(t)-W_{n}(\bar{t})\right|\right. \\
& +\int_{\tau}^{s}\left(|A T(t-r)-A T(\bar{t}-r)|\left|U_{n}(\bar{t}-r)\right|\right. \\
& \left.+|A T(t-r)|\left|U_{n}(t, r)-U_{n}(\bar{t}, r)\right|\right) d r \\
& +\int_{\tau}^{s}\left(|T(t-r)-T(\bar{t}-r)|\left|V_{n}(\bar{t}-r)\right|\right. \\
& \left.\left.+|T(t-r)-I|\left|V_{n}(t, r)-V_{n}(\bar{t}, r)\right|\right) d r\right\} \\
& \leq \alpha_{n+1} C^{n}\left\{(2 M+1) \varphi_{1}\left(\frac{t-\bar{t}}{\bar{t}-\tau}\right)\right. \\
& \left.+\int_{\tau}^{s}(2 M+1) \varphi_{1}\left(\frac{t-\bar{t}}{\bar{t}-r}\right)(\bar{t}-r)^{-1} \varphi_{n-1}\left(\frac{\bar{t}-r}{r-\tau}\right) d r\right\} \\
& \leq \alpha_{n+1} C^{n+1} \\
& \cdot 2^{-1}\left\{\varphi_{1}\left(\frac{t-\bar{t}}{\bar{t}-\tau}\right)+\int_{\tau}^{s} \varphi_{1}\left(\frac{t-\bar{t}}{\bar{t}-r}\right)(\bar{t}-r)^{-1} \varphi_{n}\left(\frac{\bar{t}-r}{r-\tau}\right) d r\right\},
\end{aligned}
$$

with $(t-\bar{t}) /(t-r) \leq \varphi_{1}((t-\bar{t}) /(\bar{t}-r))$ by $(15)$. The transformation $r=\tau+(s-\tau) \sigma$ yields

$$
\begin{aligned}
& \int_{\tau}^{s} \varphi_{1}\left(\frac{t-\bar{t}}{\bar{t}-r}\right)(\bar{t}-r)^{-1} \varphi_{n}\left(\frac{\bar{t}-r}{r-\tau}\right) d r \\
& \quad=\int_{0}^{1} \varphi_{n}\left(\frac{a+1-\sigma}{\sigma}\right) \varphi_{1}\left(\frac{b}{a+1-\sigma}\right) \frac{d \sigma}{a+1-\sigma}=q,
\end{aligned}
$$

where $a=(\bar{t}-s) /(s-\tau)$ and $b=(t-\bar{t}) /(s-\tau)$. (15) and Claim 4(iv) imply

$$
\begin{aligned}
q & \leq \int_{0}^{1} \frac{b}{a+1-\sigma} \varphi_{n}\left(\frac{a+1-\sigma}{\sigma}\right) \frac{d \sigma}{a+1-\sigma} \leq \frac{b}{a} \int_{0}^{1} \varphi_{n}\left(\frac{1-\sigma}{\sigma}\right) \frac{a}{a+1-\sigma} \frac{d \sigma}{1-\sigma} \\
& \leq \frac{b}{a} \int_{0}^{1} \varphi_{n}\left(\frac{1-\sigma}{\sigma}\right) \varphi_{1}\left(\frac{a}{1-\sigma}\right) \frac{d \sigma}{1-\sigma}=\frac{t-\bar{t}}{\bar{t}-s} \varphi_{n+1}\left(\frac{\bar{t}-s}{s-\tau}\right),
\end{aligned}
$$

and from this in turn (30) for $n+1$ follows by Claim 4(ii).

Returning to $R_{n}(t)$, Claim 5 yields the desired estimate.

Claim 6. For each $n \in N$ we have

$$
\left|R_{n}(t)\right| \leq C^{n+1} \eta^{n}\left\{1+2 \int_{0}^{1} \varphi_{n}\left(\frac{1-\sigma}{\sigma}\right) \frac{d \sigma}{1-\sigma}\right\} .
$$

PROOF. (25), (28) and (29) yield by means of (20)

$$
\begin{aligned}
\left|Z_{n}(t)\right| & \leq(M+1) C^{n} \alpha_{n}\left\{2 \int_{\tau}^{t}(t-s)^{-1} \varphi_{n}\left(\frac{t-s}{s-\tau}\right) d s+1\right\} \\
& \leq C^{n+1} \alpha_{n}\left\{1+2 \int_{0}^{1} \varphi_{n}\left(\frac{1-\sigma}{\sigma}\right) \frac{d \sigma}{\sigma}\right\}
\end{aligned}
$$

and therefore integration over $\tau_{1}, \ldots, \tau_{n}$ implies (32). 
(d) Thus it remains to derive estimates for the integrals over $\varphi_{n}$ in (32). For this purpose we consider the singular integral equation

$$
\varphi(t)=\log (1+t)+\rho \int_{0}^{1} \varphi\left(\frac{s}{1-s}\right) \log \left(1+\frac{t}{s}\right) \frac{d s}{s} .
$$

Suppose $\varphi \in C\left(\mathbf{R}_{+}, \mathbf{R}_{+}\right)$is a solution of (33) such that $\varphi(t) \leq c \sqrt{t}$. Then it is easy to see by induction that $\varphi(t) \geq \sum_{0}^{n} \rho^{j} \varphi_{j}(t)$ for each $n \in N$, hence

$$
\begin{aligned}
\sum_{0}^{\infty} \rho^{n} \int_{0}^{1} \varphi_{n}\left(\frac{s}{1-s}\right) \frac{d s}{s} & =\int_{0}^{1} \sum_{0}^{\infty} \rho^{n} \varphi_{n}\left(\frac{s}{1-s}\right) \frac{d s}{s} \leq \int_{0}^{1} \varphi\left(\frac{s}{1-s}\right) \frac{d s}{s} \\
& \leq c \int_{0}^{1} s^{-1 / 2}(1-s)^{-1 / 2} d s=c \pi<\infty
\end{aligned}
$$

i.e. with $\rho=C \eta$ we obtain

$$
\left|S_{1 \varepsilon}(t)\right| \leq 2 C\left((1-\rho)^{-1}+c \pi\right)=M_{1}<\infty \quad \text { for all } t \geq 0, \varepsilon>0
$$

provided $\eta_{0}$ is sufficiently small such that $\rho<1$. This is the uniform estimate for the $S_{1 \varepsilon}(t)$. Hence it remains to prove

Claim 7. Let $\rho>0$ be sufficiently small. Then (33) has a solution $\varphi \in$ $C\left(\mathbf{R}_{+}, \mathbf{R}_{+}\right)$such that

$$
\varphi(t) \leq c \sqrt{t} \text { for } t \geq 0, \text { and some } c>0 .
$$

ProOF. Let $Z$ denote the Banach space of all functions $\psi \in C\left(\mathbf{R}_{+}, \mathbf{R}\right)$ such that (35) holds, with norm $\|\psi\|=\sup _{t \geq 0}|\psi(t)| t^{-1 / 2}$, and $T: Z \rightarrow Z$ the linear operator

$$
(T \psi)(t)=\int_{0}^{1} \psi\left(\frac{s}{1-s}\right) \log \left(1+\frac{t}{s}\right) \frac{d s}{s}, \quad t \geq 0
$$

Since $\varphi_{1}(t)=\log (1+t)$ belongs to $Z$, we only have to show that $T: Z \rightarrow Z$ is bounded. For this purpose let $\psi \in Z$ be fixed. Then we have

$$
\begin{aligned}
|T \psi(t)| & \leq \int_{0}^{1}\left|\psi\left(\frac{s}{1-s}\right)\right| \log \left(1+\frac{t}{s}\right) \frac{d s}{s} \\
& \leq\|\psi\| \int_{0}^{1} s^{-1 / 2}(1-s)^{-1 / 2} \log \left(1+\frac{t}{s}\right) d s
\end{aligned}
$$

(15) with $\beta=1 / 2$ yields

$$
\int_{1 / 2}^{1} s^{-1 / 2}(1-s)^{-1 / 2} \log \left(1+\frac{t}{s}\right) d s \leq 2 \sqrt{t} \int_{1 / 2}^{1} s^{-1}(1-s)^{-1 / 2} d s \leq 4 \sqrt{2} \sqrt{t}
$$

For $t \geq 1 / 2,(15)$ with $\beta=1 / 4$ yields

$$
\begin{aligned}
\int_{0}^{1 / 2} s^{-1 / 2}(1-s)^{-1 / 2} \log \left(1+\frac{t}{s}\right) d s & \leq 4 \sqrt[4]{t} \int_{0}^{1 / 2} s^{-3 / 4}(1-s)^{-1 / 2} d s \\
& \leq 4 \sqrt{2} \sqrt[4]{t} \int_{0}^{1 / 2} s^{-3 / 4} d s \leq 16 \sqrt{2} \sqrt{t}
\end{aligned}
$$

since $\sqrt[4]{t} \leq \sqrt[4]{2} \sqrt{t}$ for $t \geq 1 / 2$ holds. 
For $t \leq 1 / 2,(15)$ with $\beta=1$ yields

$$
\int_{t}^{1 / 2} s^{-1 / 2}(1-s)^{-1 / 2} \log \left(1+\frac{t}{s}\right) d s \leq \sqrt{2} t \int_{t}^{1 / 2} s^{-3 / 2} d s \leq 2 \sqrt{2} \sqrt{t}
$$

and with (15), $\beta=1 / 4$ we finally get

$$
\int_{0}^{t} s^{-1 / 2}(1-s)^{-1 / 2} \log \left(1+\frac{t}{s}\right) d s \leq 4 \sqrt{2} \sqrt[4]{t} \int_{0}^{t} s^{-3 / 4} d s=16 \sqrt{2} \sqrt{t} .
$$

These estimates show that $\|T \psi\| \leq 38 \sqrt{2}\|\psi\|$, i.e. $T$ is bounded in $Z$.

(e) It is now an easy task to derive estimates for $\left|S_{\varepsilon}(t)\right|$ and $\left|S_{\varepsilon}^{\prime}(t) A^{-1}\right|$ from (34). In fact,

$$
S_{\varepsilon}^{\prime}(t) A^{-1}=S_{1 \varepsilon}(t)+\left(K_{\varepsilon} * S_{1 \varepsilon}\right)(t) .
$$

Hence

$$
\left|S_{\varepsilon}^{\prime}(t) A^{-1}\right| \leq\left|S_{1 \varepsilon}(t)\right|+\left|K_{\varepsilon}\right|_{L^{1}} \cdot \sup \left|S_{1 \varepsilon}(\tau)\right| \leq M_{1}(1+\eta) .
$$

Since

$$
S_{\varepsilon}+S_{\varepsilon} * K_{\varepsilon}=S_{\varepsilon}^{\prime} A^{-1}=S_{1 \varepsilon}+K_{\varepsilon} * S_{1 \varepsilon}
$$

holds, we get also

$$
\begin{aligned}
\sup _{t \geq 0}\left|S_{\varepsilon}(t)\right| & \leq\left(1-\left|K_{\varepsilon}\right|_{L^{1}}\right)^{-1}\left(1+\left|K_{\varepsilon}\right|_{L^{1}}\right) \sup \left|S_{1 \varepsilon}(t)\right| \\
& \leq(1+\eta)^{2}(1-\eta)^{-1} M_{1},
\end{aligned}
$$

note that all bounds are independent of $\varepsilon>0$.

Summarizing we have

Claim 8. There is a constant $M_{2}$ independent of $\varepsilon$, such that

$$
\left|S_{\varepsilon}(t)\right|+\left|S_{1 \varepsilon}(t)\right|+\left|S_{\varepsilon}^{\prime}(t) A^{-1}\right| \leq M_{2} \text { for all } t \geq 0 .
$$

(f) We are in position now to complete the proof of Step 2. Since $\left|S_{\varepsilon}(t)\right| \leq M_{2}$, its Laplace transform satisfies

$$
\left|L_{\lambda}^{n} \widehat{S_{\varepsilon}}(\lambda)\right| \leq M_{1}(\operatorname{Re} \lambda)^{-(n+1)} \quad \text { for } \operatorname{Re} \lambda>0, n \in N_{0},
$$

where $L_{\lambda}^{n}=(-1)^{n}(n !)^{-1}(d / d \lambda)^{n}$. We have

$$
\begin{aligned}
\widehat{S_{\varepsilon}}(\lambda)-H(\lambda) & =\left(\lambda-A-\widehat{K_{\varepsilon}}(\lambda) A\right)^{-1}-(\lambda-A-\widehat{d K}(\lambda) A)^{-1} \\
& =\widehat{S_{\varepsilon}}(\lambda)\left(\lambda-A-\widehat{d K}(\lambda) A-\lambda+A+\widehat{K_{\varepsilon}}(\lambda) A\right) H(\lambda) \\
& =\widehat{S_{\varepsilon}}(\lambda)\left((I-\varepsilon A)^{-1} \widehat{\rho_{\varepsilon}}(\lambda)-I\right) \widehat{d K}(\lambda) A H(\lambda),
\end{aligned}
$$

since $\widehat{K_{\varepsilon}}(\lambda)=(I-\varepsilon A)^{-1} \widehat{\rho_{\varepsilon}}(\lambda) \widehat{d K}(\lambda)$; as $\varepsilon \rightarrow 0$, the $\widehat{\rho_{\varepsilon}}(\lambda)$ converge to 1 , uniformly on compact subsets of $\operatorname{Re} \lambda>0$, and $(I-\varepsilon A)^{-1} x \rightarrow x$ for each $x \in X$. Thus $\widehat{S_{\varepsilon}}(\lambda) x \rightarrow H(\lambda) x$ as $\varepsilon \rightarrow 0$, for each fixed $x \in X$, uniformly on compact subsets of $\operatorname{Re} \lambda>0$; since $\widehat{S_{\varepsilon}}(\lambda) x$ and $H(\lambda) x$ are both holomorphic for $\operatorname{Re} \lambda>0$ this implies also $L_{\lambda}^{n} \widehat{S_{\varepsilon}}(\lambda) x \rightarrow L_{\lambda}^{n} H(\lambda) x$ for each $x \in X$ and $\operatorname{Re} \lambda>0$. Hence (37) yields

$$
\left|L_{\lambda}^{n} H(\lambda)\right| \leq M_{1}(\operatorname{Re} \lambda)^{-(n+1)} \text { for } \operatorname{Re} \lambda>0, n \in N_{0} .
$$

Now, since $H(\lambda)$ is already known to be the Laplace transform of $S \in$ $L^{1}\left(\mathbf{R}_{+}, \mathbf{B}(X)\right)$, the Widder-Post formula (see [12, Theorem 6.3.5])

$$
S(t)=\lim _{n \rightarrow \infty}\left(\frac{n}{t}\right)^{n+1} L_{n / t}^{n} H\left(\frac{n}{t}\right) \quad \text { for a.a. } t \geq 0
$$


shows that $|S(t)| \leq M_{1}$ for a.a. $t \geq 0$, i.e. $S \in L^{\infty}\left(\mathbf{R}_{+}, \mathbf{B}(X)\right)$. Similarly, one also obtains $S^{\prime} A^{-1}, S_{1} \in L^{\infty}\left(\mathbf{R}_{+}, \mathbf{B}(X)\right)$. This completes the proof of Step 2 .

STEP 3. $S(t)$ has the continuity properties asserted in Theorem 1.

Proof. For $x \in D(A)$ we have

$$
S(t) x=x+\int_{0}^{t} S^{\prime}(\tau) x d \tau
$$

Hence $S(t) x$ is continuous for each $x \in D(A)$. Since $D(A)$ is dense in $X$ and $S(t)$ is uniformly bounded, this implies the continuity of $S(t) x$ for each $x \in X$. To prove the strong continuity of $S_{1}(t)=A S(t) A^{-1}$ we consider

$$
S_{1 \varepsilon}(t)=\sum_{n=0}^{\infty}\left(A T * K_{\varepsilon}\right)^{* n} * T=\sum_{n=0}^{\infty} R_{n \varepsilon}(t)
$$

again. Since we know already that the series is absolutely and uniformly convergent w.r.t. $t \geq 0$ and $\varepsilon>0$, it suffices to show that each term $R_{n \varepsilon}(t)$ is strongly continuous, uniformly w.r.t. $\varepsilon>0$. This can be achieved by means of estimates (28)-(31) proved in Step 2 of this proof. In fact, using the notations introduced there, we observe first that

$$
\begin{aligned}
Z_{n}(t)-Z_{n}(\bar{t})= & \int_{\bar{t}}^{t}\left[A T(t-s) U_{n}(t, s) d s+(T(t-s)-I) V_{n}(t, s)\right] d s \\
& +\int_{\tau}^{\bar{t}}\left[(A T(t-s)-A T(\bar{t}-s)) U_{n}(\bar{t}, s)\right. \\
& \left.+A T(t-s)\left(U_{n}(t, s)-U_{n}(\bar{t}, s)\right)\right] d s \\
& +\int_{\tau}^{\bar{t}}\left[(T(t-s)-T(\bar{t}-s)) V_{n}(\bar{t}, s)\right. \\
& \left.+(T(t-\tau)-T(\bar{t}-\tau)) W_{n}(t)+(T(\bar{t}-\tau)-I)\left(V_{n}(t, s)-V_{n}(\bar{t}, s)\right)\right] d s
\end{aligned}
$$

for $t>\bar{t}>\tau$ implies by (28)-(31) and (13), as well as by Claim 4

$$
\begin{aligned}
& P\left|Z_{n}(t)-Z_{n}(\bar{t})\right| \leq(2 M+1) \alpha_{n} C^{n}\left\{\int_{\bar{t}}^{t}(t-s)^{-1} \varphi_{n}\left(\frac{t-s}{s-\tau}\right) d s+\varphi_{1}\left(\frac{t-\bar{t}}{\bar{t}-\tau}\right)\right. \\
&+\int_{\tau}^{\bar{t}} \frac{t-\bar{t}}{(t-s)(\bar{t}-s)} \varphi_{n}\left(\frac{\bar{t}-s}{s-\tau}\right) d s \\
&\left.+\int_{\tau}^{\bar{t}}(\bar{t}-s)^{-1} \varphi_{1}\left(\frac{t-\bar{t}}{\bar{t}-s}\right) \varphi_{n-1}\left(\frac{\bar{t}-s}{s-\tau}\right) d s\right\} \\
& \leq C_{0} \alpha_{n} C^{n} \varphi_{n+1}\left(\frac{t-\bar{t}}{\bar{t}-\tau}\right), \quad \text { where } C_{0} \geq(2 M+1)\left(3+(\log 2)^{-1}\right) .
\end{aligned}
$$

Therefore, we obtain by integration

$$
\begin{aligned}
R_{n \varepsilon}(t)-R_{n \varepsilon}(\bar{t}) & =\int \cdots \int_{D_{t}} \ldots Z_{n}(t) d \tau_{1} \cdots d \tau_{n}-\int \ldots Z_{D_{\bar{t}}} \ldots Z_{n}(\bar{t}) d \tau_{1} \cdots d \tau_{n} \\
& =\int_{D_{t} \backslash D_{\bar{t}}} \cdots \int_{D_{\bar{t}}} Z_{n}(t) d \tau_{1} \cdots d \tau_{n}+\int_{\cdots} \ldots \int\left(Z_{n}(t)-Z_{n}(\bar{t})\right) d \tau_{1} \cdots d \tau_{n}
\end{aligned}
$$


where $D_{t}=\left\{\left(\tau_{1}, \ldots, \tau_{n}\right): 0 \leq \tau_{j} \leq t-\sum_{j+1}^{n} \tau_{j}, j=1, \ldots, n\right\}$. Since $Z_{n}(t)$ is bounded by $C^{n+1} \alpha_{n} \cdot C_{n}$ for some $C_{n} \geq C_{0}$ this yields

$$
\begin{aligned}
& \left|R_{n \varepsilon}(t)-R_{n \varepsilon}(\bar{t})\right| \leq C_{n} C^{n+1}\left\{\int_{D_{t} \backslash D_{\bar{t}}} \ldots \int \alpha_{n} d \tau_{1} \cdots d \tau_{n}\right. \\
& +\int_{D_{\bar{t}-h}} \cdots \int \alpha_{n} \varphi_{n+1}\left(\frac{t-\bar{t}}{\bar{t}-\tau}\right) d \tau_{1} \cdots d \tau_{n} \\
& \left.+\int_{D_{\bar{t}} \backslash D_{\bar{t}-h}} \cdots \int \alpha_{n} d \tau_{1} \cdots d \tau_{n}\right\} \\
& \leq C_{n} C^{n+1}\left\{b_{n}(t)-b_{n}(\bar{t})+\varphi_{n+1}\left(\frac{t-\bar{t}}{h}\right) b(\infty)^{n}+\left(b_{n}(\bar{t})-b_{n}(\bar{t}-h)\right)\right\},
\end{aligned}
$$

where $b_{n}(t)=(d b)^{* n-1} * b(t)$. This estimate shows that $R_{n \varepsilon}(t)$ is left-continuous in $\mathbf{B}(X)$ and right-continuous in $\mathbf{B}(X)$ at each point of continuity of $b_{n}(t)$, uniformly w.r.t. $\varepsilon>0$. Since $R_{n \varepsilon} \rightarrow R_{n}$ in $L^{1}\left(\mathbf{R}_{+}, \mathbf{B}(X)\right)$ this implies that $R_{n}(t)$ has the same continuity properties and therefore $S_{1}(t)$ is left-continuous in $\mathbf{B}(X)$ and rightcontinuous in $\mathbf{B}(X)$ at each point of continuity of the resolvent kernel $r$ of $b$, i.e. of the solution of $r=b+d b * r$, which belongs to $\mathrm{BV}\left(\mathbf{R}_{+}, \mathbf{R}_{+}\right)$too. Note that $R_{n}(t)$ is $\mathbf{B}(X)$-continuous at $t=0$. The relation

$$
S+(S-I) * d K=S_{1}+d K *\left(S_{1}-I\right)
$$

now implies that $S(t)$ has the same continuity properties as $S_{1}(t)$ in $\mathbf{B}(X)$, and so (ii) and (iii) of Theorem 1 are proved.

It remains to show that $S_{1}(t)$ is strongly continuous from the right at the at most countable many exceptional points. For this it suffices to show that $(S-I) * d f$ is continuous for each $f \in \operatorname{BV}(J, X)$; the left-hand side of (40) is then strongly continuous on $J$, hence $S_{1}$ too. So let $f \in \mathrm{BV}(J, X)$ be given and let

$$
f(t)=f_{s}(t)+f_{c}(t)=\sum_{i} f_{i} h_{0}\left(t-t_{j}\right)+f_{c}(t)
$$

denote its decomposition into jump part and continuous part; then

$$
(S-I) * d f(t)=\sum_{i}\left(S\left(t-t_{i}\right)-I\right) f_{i}+f_{c}(0)-f_{c}(t)+\left(S * d f_{c}\right)(t) .
$$

Since $S(t)$ is strongly continuous on $\mathbf{R}_{+}$and $\sum_{i}\left|f_{i}\right|<\infty$ the first term on the right-hand side of $(41)$ is continuous, the second is so by definition, and the third one also is. In fact, $S(t)$ is $\mathbf{B}(X)$-continuous except for the set $N_{r}$, but the (vectorvalued) measure of $N_{r}$ induced by $f_{c}$ is zero, and from this continuity of $S * d f_{c}$ follows easily.

The proof is now complete.

PROOF OF THEOREM 4. We have just seen that for $f \in \operatorname{BV}(J, X)$ the function $V=(S-I) * d f$ belongs to $C(J, X)$. This shows that $u=S * f$ belongs to $W^{1, \infty}(J, X)$ and $u^{\prime}(t)=v(t)+f(t)+(S(t)-I) f(0)$, i.e. $u^{\prime}(t)-f(t)$ is continuous, 
hence the asserted continuity properties of $u^{\prime}$ follow. If $L \in \mathrm{BV}_{\text {loc }}\left(\mathbf{R}_{+}, \mathbf{B}(Y, X)\right)$ denotes the solution of $L=K+d K * L$ the relation

$$
u=A^{-1}\left(u^{\prime}-f\right)-A^{-1} d L *\left(u^{\prime}-f\right)
$$

finally shows $u \in C(J, Y)$, i.e. $u$ is a solution of $(1)$ in the sense of Definition 1.

5. Examples and applications. (i) As a generalization of (2) we consider the parabolic initial-boundary value problem of order $2 m$ :

$$
\begin{aligned}
& u_{t}(t, x)=-A(x, D) u(t, x)+\int_{0}^{t} d_{\tau} B(\tau, x, D) u(t-\tau, x)+f(t, x), \quad t \geq 0, x \in \Omega, \\
& C_{j}(x, D) u(t, x)=0, \quad x \in \partial \Omega, j=1, \ldots, m, \\
& u(0, x)=u_{0}(x), \quad x \in \Omega .
\end{aligned}
$$

Here $\Omega \subset \mathbf{R}^{N}$ denotes a bounded domain with smooth boundary $\partial \Omega$,

$$
A(x, D)=\sum_{|k| \leq 2 m} a_{k}(x) D^{k}
$$

a uniformly strongly elliptic differential operator of order $2 m$ with smooth coefficients,

$$
B(t, x, D)=\sum_{|k| \leq 2 m} b_{k}(t, x) D^{k}
$$

an arbitrary differential operator of order $2 m$ with bounded coefficients, and

$$
C_{j}(x, D)=\sum_{|k| \leq m_{j}} c_{k_{j}}(x) D^{k}, \quad j=1, \ldots, m, m_{j} \leq 2 m-1,
$$

normal boundary operators with smooth coefficients, where we used the notation $D=\left(\partial / \partial x_{1}, \ldots, \partial / \partial x_{N}\right), k=\left(k_{1}, \ldots, k_{N}\right) \in \mathbf{N}_{0}^{N},|k|=k_{1}+\cdots+k_{N}, D^{k}=$ $\left(\left(\partial / \partial x_{1}\right)^{k_{1}}, \ldots,\left(\partial / \partial x_{N}\right)^{k_{N}}\right)$.

Suppose the boundary value problem $\left(A,\left\{C_{j}\right\}_{1}^{m}, \Omega\right)$ is regular and the strong complementary condition holds (see Friedman [7, §II.19]). Then the operator $A$ in $X=L^{P}(\Omega), p \in(1, \infty)$ defined by

$$
D(A)=\left\{u \in W^{2 m, p}(\Omega): C_{j}(t, x, D) u=0, x \in \partial \Omega, j=1, \ldots, m\right\}
$$

and $(A u)(x)=A(x, D) u(x)$ generates an analytic $C_{0}$ semigroup in $X$. Let the family $\{B(t)\}_{t \geq 0}$ be defined according to

$$
(B(t) u)(x)=B(t, x, D) u(x) \text { for } u \in D(B(t))=W^{2 m, p}(\Omega) .
$$

Then $B(t)$ is closable and satisfies $D(B(t)) \supset D(A)$ for each $t \geq 0$. If the coefficients $b_{k}(t, x)$ of $B(t, x, D)$ satisfy

$$
\left|b_{k}(t, x)-b_{k}(s, x)\right| \leq b(t)-b(s), \quad x \in \Omega, t>s,
$$

for some $b \in \mathrm{BV}_{\text {loc }}\left(\mathbf{R}_{+}\right)$such that $b(0)=b(0+)$ and $b$ leftcontinuous, then Condition (B) of $\S 2$ is satisfied; equivalently this means $b_{k} \in \mathrm{BV}$ loc $\left(\mathbf{R}_{+}, L^{\infty}(\Omega)\right)$ for $|k| \leq 2 m$.

Theorem 1 implies the existence of a resolvent operator for $(42)$ in $L^{p}(\Omega)$ which is also continuous in $\mathbf{B}\left(L^{P}(\Omega)\right)$ on $(0, \infty) \backslash N_{r}$, and so in particular (42) is well 
posed in $L^{p}(\Omega)$. By Theorems 2 and 4 the solution of $(41)$ with $u_{0}=0$ belongs to $C\left(\mathbf{R}_{+}, W^{2 m, p}(\Omega)\right) \cap C^{1}\left(\mathbf{R}_{+}, L^{p}(\Omega)\right)$ at least in case

$$
f \in C_{\mathrm{loc}}^{\alpha}\left(\mathbf{R}_{+}, L^{p}(\Omega)\right) \cup\left[\mathrm{BV}_{\mathrm{loc}}\left(\mathbf{R}_{+}, L^{p}(\Omega)\right) \cap C\left(\mathbf{R}_{+}, L^{p}(\Omega)\right)\right] \cup C\left(\mathbf{R}_{+}, h_{p}^{\alpha}(\Omega)\right)
$$

for some $\alpha \in(0,1 / p)$, since for these $\alpha$ we have

$$
D_{A}\left(\frac{\alpha}{2 m}\right)=h_{p}^{\alpha}(\Omega)=\left\{u \in L^{p}(\Omega):|h|^{-\alpha}|u(\cdot+h)-u(\cdot)|_{p} \rightarrow 0\right\}
$$

see Da Prato and Grisvard $[4, \S 6]$. The solution of (42) with $f \equiv 0$ belongs to $C\left(\mathbf{R}_{+}, L^{p}(\Omega)\right)$ in case $u_{0} \in L^{p}(\Omega)$ and to $C\left(\mathbf{R}_{+}, W^{2 m, p}(\Omega)\right) \cap W_{\text {loc }}^{1, \infty}\left(\mathbf{R}_{+}, L^{p}(\Omega)\right)$ if $u_{0} \in D(A)$.

(ii) In population dynamics the following system frequently occurs by linearization; see e.g. Cushing [3].

$$
u_{j t}(t, x)=d_{j} \Delta u_{j}(t, x)+\sum_{l=1}^{n} \int_{0}^{t} d_{\tau} B_{j l}(\tau, x, D) u_{l}(t-\tau, x), \quad t \geq 0, x \in \Omega
$$

$$
\begin{aligned}
& \frac{\partial u_{j}}{\partial \nu}(t, x)=0, \quad t \geq 0, x \in \partial \Omega, j=1, \ldots, n, \\
& u_{j}(0, x)=u_{j 0}(x),
\end{aligned}
$$

where $\Omega \subset \mathbf{R}^{N}$ denotes a bounded domain again, $\Delta$ the Laplacian, $d_{j}>0, \nu(x)$ the outer normal at $\partial \Omega$, and

$$
B_{j l}(t, x, D)=b_{j l}^{0}(t, x)+\sum_{k=1}^{N} b_{j l}^{k}(t, x) \frac{\partial}{\partial x_{k}}+b_{j l}(t, x) \Delta
$$

differential operators of at most second order.

It is known that the Laplacian with no flux boundary conditions generates an analytic semigroup $e^{\Delta t}$ in $C(\bar{\Omega})$, where

$$
D(\Delta)=\left\{u \in W^{2, N+1}(\Omega): \Delta u \in C(\bar{\Omega}), \frac{\partial u}{\partial \nu}=0 \text { on } \partial \Omega\right\}
$$

see Stewart [17]. If we let $X=C(\bar{\Omega})$ and $A=\operatorname{diag}\left(d_{1} \Delta, \ldots, d_{n} \Delta\right)$ with $D(A)=$ $D(\Delta)^{n}$ then $A$ generates the analytic semigroup $e^{A t}=\operatorname{diag}\left(e^{\Delta d_{1} t}, \ldots, e^{\Delta d_{n} t}\right)$ in $X$. Condition (B) is satisfied in case

$$
\begin{aligned}
& b_{j l}^{k} \in \mathrm{BV}_{\mathrm{loc}}\left(\mathbf{R}_{+}, C(\bar{\Omega})\right) \quad \text { for } 0 \leq j, l \leq n, 0 \leq k \leq n, \\
& b_{j l} \in \mathrm{BV}_{\mathrm{loc}}\left(\mathbf{R}_{+}, C(\Omega)\right), \quad b_{j l}(0, x)=b_{j l}(0+, x)=0,
\end{aligned}
$$

holds. Theorem 1 then implies existence of a resolvent operator for (43) in $X=$ $C(\bar{\Omega})^{n}$, hence $(43)$ is well posed in this space.

It should be noted that here we cannot admit $B_{j l}$ to be any second order operator with coefficients in $\mathrm{BV}_{\text {loc }}\left(\mathbf{R}_{+}, C(\bar{\Omega})\right)$ since the domain relation $D(B(t)) \supset D(A)$ will in general not be fulfilled, as $D(\Delta) \supsetneqq\left\{u \in C^{2}(\Omega): \partial u / \partial \nu=0\right.$ on $\left.\partial \Omega\right\}$. This is one of the pitfalls when working in the space of continuous functions.

(iii) Example (ii) can be used to illustrate the remark following Theorem 1. For this purpose let $n=1, b_{11}^{k}=0$ and $b_{11}(t, x)=b(x) h_{0}(t-1)$ with $b(x)$ continuous; for $t \in(1,2]$ the resolvent $S(t)$ then is given by

$$
S(t)=e^{\Delta t}+\int_{1}^{t} e^{\Delta(t-s)} b(x) \Delta e^{\Delta(s-1)} d s
$$


Hence, if we let $\mu_{0}(x)$ denote the solution of the Neumann problem

$$
\Delta \mu_{0}=1 \text { in } \Omega, \partial \mu_{0} / \partial \nu=0 \text { on } \partial \Omega, \text { then } A S(t) \mu_{0}(x)=1+e^{\Delta(t-1)} b(x)-b(x)
$$

which is as regular as $b(x)$. In particular, $S^{\prime}(t) \mu_{0}, A S(t) \mu_{0} \notin Y_{\alpha}$ for any $\alpha \in(0,1)$ if $b(x)$ is only continuous.

\section{REFERENCES}

1. R. M. Christensen, Theory of viscoelasticity: An introduction, Academic Press, New York, 1971.

2. B. D. Coleman and M. E. Gurtin, Equipresence and constitutive equations for heat conductors, Z. Angew. Math. Phys. 18 (1967), 199-208.

3. J. Cushing, Integrodifferential equations and delay models in population dynamics, Lecture Note in Biomath., vol. 20, Springer-Verlag, Heidelberg, 1977.

4. G. Da Prato and P. Grisvard, Equations d'evolution abstraites non lineaires de type parabolique, Ann. Mat. Pura Appl. 120 (1979), 329-396.

5. G. Da Prato and M. Ianelli, Existence and regularity for a class of integrodifferential equations of parabolic type, preprint.

6. G. Da Prato, M. Ianelli and E. Sinestrari, Regularity of solutions of a class of linear integrodifferential equations in Banach spaces, J. Integral Equations 8 (1985), 27-40.

7. A Friedman, Partial differential equations, Holt, Rinehart and Winston, New York, 1969.

8. A. Friedman and M. Shinbrot, Volterra integral equations in Banach spaces, Trans. Amer. Math. Soc. 126 (1967), 131-179.

9. R. Grimmer and F. Kappel, Series expansions for resolvents of Volterra integrodifferential equations in Banach space, SIAM J. Math. Anal. 15 (1984), 595-604.

10. R. Grimmer and A. J. Pritchard, Analytic resolvent operators for integral equations in Banach spaces, J. Differential Equations 50 (1983), 234-259.

11. R. Grimmer and J. Prüss, On linear Volterra equations in Banach spaces, Comput. Math. Appl. 11 (1985), 189-205.

12. E. Hille and R. S. Phillips, Semigroups and functional analysis, Amer. Math. Soc. Colloq. Publ., vol. 31, Amer. Math. Soc., Providence, R.I., 1957.

13. R. S. Phillips, A note on the abstract Cauchy problem, Proc. Nat. Acad. Sci. U.S.A. 40 (1954), 244-248.

14. J. Prüss, Lineare Volterra Gleichungen in Banachräumen, Habilitationsschrift, Paderborn, 1984.

15. _ On bounded solutions of Volterra equations, preprint.

16. E. Sinestrari, On the abstract Cauchy problem of parabolic type in spaces of continuous functions, J. Math. Anal. Appl. 107 (1985), 16-66.

17. B. Stewart, Generation of analytic semigroups by strongly elliptic operators under general boundary conditions, Trans. Amer. Math. Soc. 259 (1980), 299-310.

18. G. F. Webb, Regularity of solutions to an abstract inhomogeneous linear differential equation, Proc. Amer. Math. Soc. 62 (1977), 271-277.

19. D. V. Widder, The Laplace transform, Princeton Univ. Press, Princeton, N.J., 1941.

FACHBereich 17, Universität-Gesamthochschule Paderborn, Warburger STr. 100, 4790 PADERBorn, Federal Republic of GERMANY 